\title{
Hamiltonian formalism for nonlinear waves
}

\author{
V.E.Zakharov ${ }^{1}$ and E.A.Kuznetsov ${ }^{2}$ \\ Landau Institute for Theoretical Physics, \\ 2 Kosygin str., Moscow 117334, Russia
}

\begin{abstract}
Hamiltonian description for nonlinear waves in plasma, hydrodynamics and magnetohydrodynamics is presented. The main attention is paid to the problem of canonical variables introducing. The connection with other approaches of the Hamiltonian structure introducing is presented, in particular, with the help of the Poisson brackets expressed in terms of natural variables. It is shown that the degeneracy of the noncanonical Poisson brackets is connected for the system of hydrodynamic type with the specific symmetry, namely, with the relabeling transformations of the Lagrangian markers of fluid particles. All known theorems about the vorticity conservation (the Ertel's, Cauchy's and Kelvin's theorems, the frosenness of vorticity and conservation of the topological Hopf invariant) are a sequence of this symmetry. The canonical variables are introduced into the collisionless plasma kinetics and into the Benney equation. The problem of Hamiltonian structures is discussed for surface and internal waves as well as for the Rossby waves. The Hamiltonian structure also is introduced for the Davey-Stuartson equation, describing the interaction of quasimonochromatic waves with the induced low-frequency medium motion. At the end of this survey a general method for investigation of weak nonlinear waves is considered, based on both the classical perturbation theory and reduction of Hamiltonians.
\end{abstract}

\section{Contents}

1 Introduction 1

2 General Remarks 5

3 Hamiltonian Formalism in Continuous Media 9

4 Canonical Variables in Hydrodynamics 12

${ }^{1}$ e-mail: zakharov@itp.ac.ru

2e-mail: kuznetso@itp.ac.ru 
5 Noncanonical Poisson Brackets

6 Ertel's Theorem 23

7 Gauge Symmetry - Relabeling Group $\quad 27$

8 The Hopf Invariant and Degeneracy of the Poisson Brackets 34

9 Inhomogeneous Fluid and Surface Waves 38

10 Hamiltonian Formalism for Plasma and Magnetohydrodynamics 44

11 The Hamiltonian Formalism in Kinetics 53

12 Classical Perturbation Theory and Reduction of Hamiltonians 57

\section{Introduction}

The equations of hydrodynamics and their generalizations are among the most basic tools for description of nonlinear waves in macroscopic physics. In studying them, an important question is whether these equations, in the case of absence of dissipation, have a Hamiltonian structure. This problem is primarily important in connection with the problem of quantization. However, also in the classical case, establishing that a given system is Hamiltonian allows one to hope (although this is not always a simple matter) to introduce explicitly canonical variables, after which all the variants of perturbation theory are considerably simplified and standardized (cf., for example, $[1,2,3,4])$. In particular, this approach gives an opportunity to consider all nonlinear processes from the general point of view without fixing their proper peculiarities connected with a given concrete medium. The Hamiltonian approach gives also certain advantages when approximations must be performed. Classical example of this is a description of well-separated space or/and time scales, in particular, of high-frequency and low-frequency waves (for review, see the remarkable book of Whitham [5]). For the Hamiltonian continuous systems, the stability problem for stationary solutions as cnoidal waves, solitons, vortices, etc. is formulated more or less at the same manner and it can be solved by studying the quadratic Hamiltonian for small perturbations or by taking the Hamiltonian with combination with another integrals (numbers of particles, momentum, etc.) as the Lyapunov functional if one treats the nonlinear stability (cf., for instance, $[6,7,8]$ ).

Besides hydrodynamics, the equations of the hydrodynamic type are widely used for description of various processes in plasma physics as well as in magnetohydrodynamics. They combine the equation of medium motion and the Maxwell equations for electromagnetic field. These models play also an essential role for solid state physics and nonlinear optics. 
The problem of the Hamiltonian structure for hydrodynamic equations has a long history. There are two traditional approaches to answer it. First, one can try, for some system or other, to directly guess a complete set of canonical variables. Then the problem of calculating Poisson brackets between any physical quantities is automatically solved, and one succeeds also in writing down a variational principle. Usually the Hamiltonian variables are expressed in terms of the natural variables (velocity, pressure) in a by no means trivial fashion.

An alternative path is to find directly expressions for the Poisson brackets in "natural" variables. This does not enable one to introduce a variational principle, but for many physical problems, including the problem of quantization, it appears to be sufficient. The equations of hydrodynamic type have the same degree of nonlinearity (quadratic in the velocities) as the energy integral. It then follows that the expression for the Poisson bracket must be linear with respect to the variables (the velocity, the density, etc.) that enter these equations. It is easy to show that all such brackets are brackets of the Berezin-Kirillov-Kostant type on certain Lie groups. This quite important fact was understood relatively recently, apparently first by V.I.Arnold [9], [10] (see also, [11]) although Poisson brackets between velocity components were already calculated in connection with the problem of quantization in a paper of L.D.Landau [12]. Also devoted to these notions were some papers of I.E.Dzyaloshinskii and G.E.Volovik [13], and S.P.Novokov. In For the equations of magnetohydrodynamics the noncanonical Poisson brackets were calculated first by Greene and Morrison [15] and for the Vlasov-Maxwell equations for a plasma they were obtained by Morrison [16].

As for canonical variables, for the ideal hydrodynamics of a homogeneous incompressible fluid they were already found in the previous century by Clebsch (cf., for example, [17]). The topological meaning of these variables was clarified in the paper of Kuznetsov and Mikhailov [18]. In 1932, H.Bateman [19], and later independently B.I.Davydov [20], extended the result of Clebsch to a compressible barotropic liquid. In 1952 for nonbarotropic flows of ideal liquid variables were found by I.M.Khalatnikov [21]. Later this result was rediscovered in a set of other articles (see, for example, [22]).

From these results one can obtain the canonical variables for an incompressible fluid of variable density, including fluids with a free boundary, as was done by Kontorovich, Kravchik and Time [23]. However, the extremely important problem from the point of view of surface waves, of the Hamiltonian description of a fluid with free surface, was solved earlier by one of the authors of the present book (V.E.Zakharov). The canonical variables were introduced without proof in 1966 [24], and the complete proof was published in 1968 in [25]. In these papers only potential flows of the fluid were considered. A partial transfer of the results to the case of nonpotential flow was accomplished by Voronovich [26] and Goncharov [27], who also solved the problem of the Hamiltonian description of internal waves in the ocean. A presentation of these results can be found in the monograph of Yu.Z.Miropolskii [28] as well as a recent 
book by Goncharov and Pavlov [29], both written entirely from the point of view of Hamiltonian formalism.

Of especial interest is the Hamiltonian formalism for the Benney equations, describing nonpotential long waves on shallow water. The system of Benney equations is completely integrable [30],[31], and the Hamiltonian formalism for them was formulated (in the language of Poisson brackets between moments of the longitudinal velocity) in a paper of Manin and Kupershmidt [32].

Canonical variables enabling one to calculate Poisson brackets between any quantities, were found for the Benney equations in [30]. This question turned out unexpectedly to be related to the question of the Hamiltonian description of plasma, which had attracted attention earlier. A Hamiltonian description of magnetohydrodynamics was achieved by the authors of the present book in 1970 [33]. Canonical variables in a two-fluid hydrodynamic model were introduced in [34], and were used later in various papers describing nonlinear processes in plasma (cf., for example [35]). This did not solve the question of introducing canonical variables in the collisionless kinetics of a plasma, although, after paper [31], it became clear that such variables must exist. In the present survey we introduce such variables, using the equivalence of the Vlasov equations to an infinite system of hydrodynamic equations. This equivalence, which was noted by one of the authors (E.A.Kuznetsov) is established by a Radon transform, and was essentially used in [30], where it was shown that the Benney equations are equivalent to one variant of the Vlasov equations.

In the present survey we also give a systematic description of the result recalled above. In addition we discuss the interesting question of Hamiltonian structure for a two-dimensional incompressible hydrodynamics, and for the Charny-ObukhovHasegawa-Mima equation describing Rossby waves. In these systems, one has not succeeded so far in introducing suitable canonical variables, although the existence of a Hamiltonian structure is a proven fact. Recently Piterbarg [39], generalizing the results of the papers [38], turned out to prove that the noncanonical Poisson brackets for such systems for arbitrary flows with closed stream lines can be reduced to the Gardner-Zakharov-Faddeev brackets appearing at first for the integrable equations [40] and to suggest a constructive scheme of canonical basis finding. Finally we consider some general properties of Hamiltonian systems with a continuous number of degrees of freedom.

The basis of this survey was the paper of the authors [1], published in 1986 in English in a sufficiently rare journal and therefore became unknown for a wide audience both Russian and in abroad. Against [1] the text of this survey is revised and broaden significantly. First of all, both revision and addition were subject for the problems of the noncanonical Poisson brackets and their degeneracy. For system of the hydrodynamic type this degeneracy is connected with a hidden symmetry of the equations, being in fact the gauge one. This symmetry has the Lagrangian origin; it relates to the group of transformations relabeling the Lagrangian variables marked each fluid particle. Evidently no changes in markers must influence on the system 
dynamics. First this fact was understood sufficiently completely by R.Salmon [41] in 1982 although Eckart in 1938 and then in 1960 [44] and later Newcomb understood the role of this symmetry. In particular, all known theorems about the vorticity conservation, i.e., the Ertel theorem about the existence of the Lagrangian (material) invariants [42] (see also [53], pp 31-32), the Cauchy theorem of frozenness of vorticity into a fluid [17] and the Kelvin theorem about the conservation of the velocity circulation (see, for instance, [53]), as well as the conservation of the topological Hopf invariant [55, 56] characterized the flow knottiness, are a consequence of this symmetry. This symmetry is connected also with introducing the canonical Clebsch variables and their gauge symmetry.

One should note that introducing canonical variables of the Clebsch kind into the systems of the hydrodynamic type allows one to find expressions for all noncanonical Poisson brackets known up to now, starting from the canonical one. This fact first was demonstrated by the authors of the given survey $[48,1]$ for the equations of ideal hydrodynamics and for the kinetic Vlasov-Maxwell equations for plasma. However, passing to the opposite direction, i.e., finding canonical brackets from the noncanonical one, in the general situation entails some difficulties, connected with the noncanonical brackets degeneracy.

In this survey we consider in more details all these questions for the hydrodynamic equations. Here we don't discuss the role of this symmetry for another models, except the MHD equations (about this subject see the recent paper [49]). Now this question for systems of the hydrodynamic type is far well studied and requires additional investigations. In our opinion, it has a principle meaning for understanding many nonlinear phenomena which take place in fluids and plasma. First of all these are the processes of reconnection of vortex lines for fluids or magnetic field lines in plasma, namely, the processes which change the system topology.

\section{General Remarks}

We recall some elementary facts. The most naive definition of a finite-dimensional Hamiltonian system reads as follows. One considers a system of an even number of differential equations for the time-dependent functions $q_{k}(t), p_{k}(t)(k=1, \ldots, N)$, having the form

$$
\dot{q}_{k}=\frac{\partial H}{\partial p_{k}}, \dot{p}_{k}=-\frac{\partial H}{\partial q_{k}} .
$$

Here $H\left(p_{1}, \ldots, p_{N}, q_{1}, \ldots, q_{N}\right)$, which is a given function of the variables, is the Hamiltonian.

The definition presented here is far from being always satisfactory, since it assumes implicitly that the domain of variation of the $p_{i}$ and $q_{i}$ (the phase space) is a domain in the real vector space $R^{2 N}$. However, already for the case of the mathematical pendulum, where the generalized coordinate is an angle, one must identify its 
values that differ by $2 \pi$. Thus the phase space of the pendulum is a cylinder, which is extremely significant, since any functions defined uniquely on the cylinder must be periodic functions of the angular coordinate. The situation becomes even more complicated when we consider the spherical pendulum or the motion of a rigid body with one point fixed. All of these examples deal with the next class of Hamiltonian systems in degree of non-triviality, where one can, with a sufficient degree of definiteness, distinguish two groups of variables-generalized coordinates $q_{1}, \ldots, q_{N}$ and generalized momenta $p_{1}, \ldots, p_{N}$. The separation is based on the fact that the generalized coordinates give a point on an arbitrary $N$-dimensional manifold (configuration space) $M$, while the momenta can have arbitrary values in the vector space of momenta $R^{N}$. In this case the phase space of the system, $G=T^{*}(M)$ is the tangent bundle of the manifold $M$. Hamiltonian systems of this type preserve the basic properties of "naive" Hamiltonian systems. In particular, the variational principle in the Hamiltonian form is valid for them and one can go over to a Lagrangian description.

Only systems of this type are usually described in the standard textbooks of theoretical physics.

It is important, however, to consider Hamiltonian systems of more general form, in which it is impossible to make a unique separation of variables into coordinates and momenta. Such systems are conveniently described in terms of generalized coordinates, that are generally speaking not canonical. Let $G$, the phase space of the system, be a manifold covered by some system of maps. We assume that on the manifold $G$ there is given a symplectic structure - a nondegenerate closed two-form $\Omega$. This means that at each point a twice covariant anti-symmetric tensor $\Omega_{i j}=-\Omega_{j i}$ is defined. Suppose that $x_{i}$ are the local coordinates of some point. The closure condition implies that $\Omega_{i j}$ obeys the system of equations

$$
\frac{\partial \Omega_{i j}}{\partial x_{k}}+\frac{\partial \Omega_{j k}}{\partial x_{i}}+\frac{\partial \Omega_{k i}}{\partial x_{j}}=0
$$

with $\operatorname{det}|| \Omega_{i j} \| \neq 0$.

A system of differential equations defined on $G$ is said to be Hamiltonian if there exists a function $H$ on $G$, so that, in the neighborhood of each point identified by $x_{i}$ one has

$$
\Omega_{i j} \dot{x}_{j}=\frac{\partial H}{\partial x_{i}} .
$$

It is easy to see that under changes of coordinates $x_{i}=x_{i}\left(\tilde{x}_{1}, \ldots, \tilde{x}_{N}\right)$, for which Jacobian $\partial\left(x_{1}, \ldots, x_{N}\right) / \partial\left(\tilde{x}_{1}, \ldots, \tilde{x}_{N}\right) \neq 0$, then the equation (2.3) remains invariant. In this case the matrix $\Omega$ transforms as follows,

$$
\tilde{\Omega}_{l m}=\frac{\partial x_{i}}{\partial \tilde{x}_{l}} \Omega_{i j} \frac{\partial x_{j}}{\partial \tilde{x}_{m}} .
$$

A manifold on which one can assign symplectic structure is said to be symplectic. It necessarily has even dimension (otherwise $\operatorname{det} \Omega_{i j}=0$ ). 
Within each simply connected region Eq.(2.2) can be integrated to read:

$$
\Omega_{i j}=\frac{\partial A_{i}}{\partial x_{j}}-\frac{\partial A_{j}}{\partial x_{i}} .
$$

where $A_{i}(x)$ are the "potentials" of the form $\Omega_{i j}$. If the solutions of the system (2.3) do not extend beyond the limits of this region, the variational principle $\delta S=0$ is valid, where

$$
S=\int\left(A_{i} \dot{x}_{i}+H\right) d t
$$

It has been noted that the variational principle (2.5) exists globally only if the form $\Omega_{i j}$ is exact, i.e., if the relation (2.4) can be extended over the whole manifold $G$. Generally speaking, $A_{i}$ are multivalued functions on $G$, that acquire a nonzero addition in going around any cycle not homologous to zero. Locally, in each simply connected region one can, by a suitable change of variables, bring the system to canonical coordinates, i.e., to the form (2.1) (Darboux's theorem). However, globally (over all $G$ ) doing this is generally not possible, even if the form (2.4) is exact. Due to the assumption of the nondegeneracy of the form $\Omega_{i j}$, Eq. (2.3) can be written in the form

$$
\dot{x}_{i}=R_{i j} \frac{\partial H}{\partial x_{j}} .
$$

Here $R_{i j}=-R_{j i}$ is the matrix reciprocal to $\Omega_{i j}$, such as $R^{-1}=\Omega$. It is then easily verified that the relations (2.2) are equivalent to the relations

$$
R_{i m} \frac{\partial}{\partial x_{m}} R_{j k}+R_{k m} \frac{\partial}{\partial x_{m}} R_{i j}+R_{j m} \frac{\partial}{\partial x_{m}} R_{i k}=0 .
$$

Next, by means of the matrix $R$ one defines the Poisson bracket between any functions $A$ and $B$ given on $G$ :

$$
\{A, B\}=\sum R_{i j} \frac{\partial A}{\partial x_{i}} \frac{\partial B}{\partial x_{j}}
$$

From the antisymmetry of $R_{i j}$ it follows that

$$
\{A, B\}=-\{B, A\}
$$

while the relations (2.7) guarantee that the Jacobi identity

$$
\{\{A, B\}, C\}+\{\{B, C\}, A\}+\{\{C, A\}, B\}=0
$$

is satisfied. Because of the nondegeneracy of the matrix $\Omega_{i j}$, in each coordinate system the matrix $R_{i j}$ is also nondegenerate. The matrix $R$ is called the symplectic operator, and it plays the same role as the metric tensor $g_{i j}$ in Eucledian geometry. 
The condition (2.7) is analogous to the vanishing of the curvature tensor for Eucledian space, and, respectively, the canonical form

$$
\Omega=\left(\begin{array}{cc}
0 & I \\
-I & 0
\end{array}\right)
$$

has the same meaning as

$$
g=I
$$

in Eucledian space.

The next step for generalizing the Hamiltonian system is to drop the requirement of nonsingularity of $R$. This variant of the Hamiltonian mechanics is called by the Poisson mechanics. If $\operatorname{det}|| R_{i k} \|=0$, then a return to the form (2.3) is impossible. Suppose that $\xi_{i}^{\alpha}(\alpha=1, \ldots, k)$ is based in the co-kernel of the operator $R_{i j}$ (i.e., $\left.\xi_{i} R_{i j}=0\right)$. Then, from (2.6), it follows the relations

$$
\xi_{i}^{\alpha} \dot{x}_{i}=0, \alpha=1, \ldots, k
$$

follow. In a one-connected domain in which rank of the matrix $R$ is constant, due to the Frobenious theorem, Eqs.(2.10) can be integrated:

$$
f^{\alpha}\left(x_{1}, \ldots, x_{n}\right)=\mathrm{const}, \alpha=1, \ldots, k .
$$

In their turn, these relations are connected with vectors $\xi_{i}^{\alpha}$ by the evident formulas:

$$
\xi_{i}^{\alpha}=\frac{\partial f^{\alpha}}{\partial x_{i}}
$$

The constants $f^{\alpha}$ are called Casimirs. Moreover, the Frobenious theorem and the relations (2.7) guarantee that all these $k$ invariants are functionally independent. They are evidently integrals of motion for our Hamiltonian system. These integrals separate $G$ into manifolds invariant under the system (2.6) (symplectic leaves). On each of them one can introduce the usual Hamiltonian mechanics. From our remarks it is clear that the possibility of introducing Poisson brackets implies the system under consideration to be Hamiltonian in the weakest sense.

Of special interest is the case where the metric elements $R_{i j}$ are linearly dependent on the coordinates as follows:

$$
R_{i j}=e_{i j, m} x_{m}
$$

From condition (2.7) it now follows that the $e_{i j, m}$ are subject to the relations

$$
e_{i k, m} e_{j m, l}+e_{j i, m} e_{k m, l}+e_{k j, m} e_{i m, l}=0
$$

i.e., they are the structure constants of some Lie algebra $L$. Calculating the bracket between quantities $x_{i}, x_{j}$, it can be checked that

$$
\left\{x_{i}, x_{j}\right\}=R_{i j}=e_{i j, m} x_{m} .
$$


Thus, the space $G$ itself is now a Lie algebra $L$. The constants $f^{\alpha}$ in this case are just the Casimirs of this Lie algebra, and they commute with any elements from the algebra,

$$
\left\{f^{\alpha}, .\right\}=0
$$

The matrix $R_{i j}$ is in general degenerate. However, the relations (2.10) are always integrable. Consider the algebra $L^{*}$, dual to $L$, and the corresponding Lie group $l^{*}$. Here the algebra $L$ forms the co-adjoint representation of the group $l^{*}$. Relations (2.10) are invariant under the action of the group $l^{*}$, and so conditions (2.12) hold, and define the orbits of the action of the group $l^{*}$ in $L$. On these orbits (cf. Kirillov [36], Kostant [37]) there exists a fully valid Hamiltonian mechanics.

If the Hamiltonian is polynomial in its variables, then the equations are also polynomials in the canonical coordinates, and they have a nonlinearity that is one degree lower. If the degree of nonlinearity of the investigated system coincides with the degree of nonlinearity of the Hamiltonian, then the matrix is linear in the coordinates, and the symplectic manifold $G$ is the orbit of some Lie group in its co-adjoint representation. This currently happens for equations of hydrodynamic type.

Another interesting case is the situation when the Poisson structure $R$ depends on coordinates $x_{i}$ quadratically. In this case it can be considered as the classical $R$ matrix which plays the important role in theory of Hamiltonian systems integrable by the inverse scatterring transform. This theory, however, is far from a scope of this survey and further we shall not touch this question.

In the next sections on the concrete examples we show how both canonical variables are introduced and matrix elements are calculated.

\section{Hamiltonian Formalism in Continuous Media}

The introduction of a Hamiltonian structure for conservative nonlinear media is essentially a generalization of the Hamiltonian formalism for systems with a finite number of degrees of freedom to systems with a continuum number of degrees of freedom. We shall basically understand this to give a description of the dynamics of waves evolving in a continuous medium by means of canonical variables. There are no general recipes for the introduction of canonical variables in continuous media. To solve this problem it is sometimes useful to make use of a Lagrangian with constraints, which one takes in the form of some equations of motion. This method, which apparently arose in the work of B.I.Davydov [20], is justified when the expression for the Lagrangian without the constraints comes directly from mechanics or field theory. Such procedure, in particular, apply to systems of hydrodynamic type that will be considered in this survey, and widely used for describing nonlinear waves in plasma, in hydrodynamics and magnetohydrodynamics. For this purpose, it will be required to determine We shall find the canonical variables for all of these systems. 
Suppose that the medium is described by a pair of canonical variables - the generalized coordinate $q(\mathbf{r}, t)$ and the generalized momentum $p(\mathbf{r}, t)$, whose evolution is given by the Hamiltonian equations:

$$
\frac{\partial p}{\partial t}=-\frac{\delta H}{\delta q}, \frac{\partial q}{\partial t}=\frac{\delta H}{\delta p}
$$

Here the Hamiltonian is some functional of $p$ and $q$. Formally it can be written as a series in powers of the canonical variables:

$$
H=\sum_{n=0}^{\infty} \sum_{k=0}^{n} \int G_{n}^{k}\left(\mathbf{r}_{1}, \ldots, \mathbf{r}_{k}, \mathbf{r}_{k+1}, \ldots, \mathbf{r}_{n}\right) p\left(\mathbf{r}_{1}\right) \ldots p\left(\mathbf{r}_{k}\right) q\left(\mathbf{r}_{k+1}\right) \ldots q\left(\mathbf{r}_{n}\right) d \mathbf{r}_{1} \ldots d \mathbf{r}_{n} .
$$

This expansion, in the absence of external forces, begins with quadratic terms in $p$ and $q$. For spatially homogeneous media the structure functions $G_{n}^{k}$ are functions of the differences $\left(\mathbf{r}_{i}-\mathbf{r}_{j}\right)$. In particular, for such media the quadratic term in the expansion $H_{0}$ has the form:

$$
H_{0}=\frac{1}{2} \int\left[A\left(\mathbf{r}-\mathbf{r}^{\prime}\right) p(\mathbf{r}) p\left(\mathbf{r}^{\prime}\right)+2 B\left(\mathbf{r}-\mathbf{r}^{\prime}\right) p(\mathbf{r}) q\left(\mathbf{r}^{\prime}\right)+C\left(\mathbf{r}-\mathbf{r}^{\prime}\right) q(\mathbf{r}) q\left(\mathbf{r}^{\prime}\right)\right] d \mathbf{r} d \mathbf{r}^{\prime}
$$

whose diagonalization solves the problem of stability of a homogeneous medium under small perturbations.

To solve it we first carry out a Fourier transformation in the coordinates

$$
\begin{aligned}
& p(\mathbf{r})=\frac{1}{(2 \pi)^{3 / 2}} \int p_{k} e^{i \mathbf{k r}} d \mathbf{k}, p_{k}=p_{-k}^{*}, \\
& q(\mathbf{r})=\frac{1}{(2 \pi)^{3 / 2}} \int q_{k} e^{i \mathbf{k r}} d \mathbf{k}, q_{k}=q_{-k}^{*},
\end{aligned}
$$

As a result, equation (3.3) is rewritten in the form

$$
H_{0}=\frac{1}{2} \int\left[A_{k} p_{k} p_{k}^{*}+2 B_{k} p_{k} q_{k}^{*}+C_{k} q_{k} q_{k}^{*}\right] d \mathbf{k} .
$$

The Fourier transforms of the structural functions that enter here have the following properties:

$$
\begin{aligned}
& A_{k}=A_{k}^{*}=A_{-k}, C_{k}=C_{k}^{*}=C_{-k}, \\
& B_{k}=B_{1 k}+i B_{2 k}=B_{1-k}-i B_{2-k} .
\end{aligned}
$$

In the $k$-representation the Eqs.(3.1) then take the form

$$
\frac{\partial p_{k}}{\partial t}=-\frac{\delta H}{\delta q_{k}^{*}}, \frac{\partial q_{k}}{\partial t}=\frac{\delta H}{\delta p_{k}^{*}}
$$


The equations for small perturbations are obtained from this by varying the Hamiltonian $H_{0}$. Analysis of these equations shows that waves can propagate in the medium with frequencies

$$
\omega_{1,2}=-B_{2 k} \pm \sqrt{A_{k} C_{k}-B_{1 k}^{2}} .
$$

The medium will be stable with respect to small perturbations if

$$
A_{k} C_{k}-B_{1 k}^{2}>0
$$

and, respectively, unstable in the opposite case. The latter case, for instance, can be realized in a cold plasma with a monochromatic electron beam when plasma electrons and beam electrons can be considered as two independent fluids.

In the following we shall assume that the stability condition (3.4) is satisfied. For media that are invariant under reflection $(B(\mathbf{r})=B(-\mathbf{r}))$, one obtains

$$
B_{2 k}=0 \text { and } \omega_{k}^{2}=A_{k} C_{k}-B_{1 k}^{2} .
$$

We further carry out a canonical transformation from the variables $p_{k}$ and $q_{k}$ to normal variables $a_{k}$ and $a_{k}^{*}$ :

$$
\begin{gathered}
p_{k}=U_{k} a_{k}+U_{k}^{*} a_{-k}^{*}, \quad\left(U_{k}=U_{-k}\right), \\
q_{k}=V_{k} a_{k}+V_{k}^{*} a_{-k}^{*}, \quad\left(V_{k}=V_{-k}\right) .
\end{gathered}
$$

in which the quadratic Hamiltonian is

$$
H_{0}=\int \omega_{k} a_{k}^{*} a_{k} d \mathbf{k}
$$

and the equations of motion have the form:

$$
\frac{\partial a_{k}}{\partial t}=-i \frac{\delta H}{\delta a_{k}^{*}} .
$$

Here $\omega_{k}$ denotes one of the functions $\omega_{1,2}$.

Substituting the transforms (3.5) into (3.3), from a comparison with (3.6) we get a system of equations for determining $U_{k}$ and $V_{k}$. By requiring that this transformation is canonical, we get

$$
U_{k} V_{k}^{*}-U_{k}^{*} V_{k}=-i
$$

and find from this system

$$
\begin{gathered}
U_{k}=i \frac{\left(B_{1 k}-i \omega_{0 k}\right)}{\sqrt{2} A_{k} \omega_{0 k}} \exp \left(i \varphi_{k}\right) \\
V_{k}=-i \sqrt{\frac{A_{k}}{2 \omega_{0 k}}} \exp \left(i \varphi_{k}\right) .
\end{gathered}
$$


In the above expressions $\omega_{o k}$ is the quantity $\operatorname{sign}\left[A_{k}\right]\left(A_{k} C_{k}-B_{1 k}^{2}\right)^{1 / 2}$, and $\varphi_{k}$ is an arbitrary phase factor, which we shall set equal to zero from now on (this corresponds to a simple redefinition of $\left.a_{k}: a_{k} \rightarrow a_{k} e^{i \varphi k}\right)$.

Let us now explicitly consider the complete frequency

$$
\omega_{k}=-B_{2 k}+\operatorname{sign}\left[A_{k}\right]\left(A_{k} C_{k}-D_{1 k}^{2}\right)^{1 / 2}
$$

that is the dispersion law for the waves. It is essential that the sign of the frequency coincides with the sign of the wave energy in the nonlinear medium. ${ }^{3}$ By this reason all waves can be divided in two big classes: waves with positive energy and waves with negative energy. All well-known waves (gravity and capillary waves on the fluid surface, acoustic and electromagnetic waves, and so on) belong to the first class. The waves with a negative energy typically appear in media with some current (it may be electron or ion beams in plasma, or flow of one fluid with respect to another, etc.) and in this case the origin of a negative frequency is connected with the Doppler effect. One should say that there is no principle difference in the nonlinear interaction between waves within their respective classes. This arises for the interaction between waves with positive and negative energies.

In order to classify the nonlinear interaction between waves, let us consider the next terms in the expansion in powers of $a$ and $a^{*}$, which can be obtained after substitution of (3.5) into (3.2). In particular, the cubic term $H_{1}$ has the form

$$
\begin{gathered}
H_{1}=\int\left(V_{k k_{1} k_{2}} a_{k}^{*} a_{k_{1}} a_{k_{2}}+\text { c.c. }\right) \delta_{k-k_{1}-k_{2}} d \mathbf{k} d \mathbf{k}_{1} d \mathbf{k}_{2} \\
+\frac{1}{3} \int\left(U_{k k_{1} k_{2}} a_{k}^{*} a_{k_{2}}^{*} a_{k_{2}}^{*}+\text { c.c. }\right) \delta_{k+k_{1}+k_{2}} d \mathbf{k} d \mathbf{k}_{1} d \mathbf{k}_{2}
\end{gathered}
$$

where the matrix elements of $U$ and $V$ have the following symmetry properties:

$$
U_{k k_{1} k_{2}}=U_{k k_{2} k_{1}}=U_{k_{2} k_{1} k}, V_{k k_{1} k_{2}}=V_{k k_{2} k_{1}}
$$

Among the fourth-order terms, we shall be interested in the term of the form

$$
H_{2}=\frac{1}{2} \int T_{k_{1} k_{2} k_{3} k_{4}} a_{k_{1}}^{*} a_{k_{2}}^{*} a_{k_{3}} a_{k_{4}} \delta_{k_{1}+k_{2}-k_{3}-k_{3}} \prod_{i} d \mathbf{k}_{i} .
$$

Each term in the expansion of $H$ in powers of $a$ and $a^{*}$ has a simple physical meaning. The equation of motion in the form (3.7) is the limit of the corresponding quantum equations for the Bose operators in the case of a classical wave field, where the variables $a^{*}$ and $a$ appear as analogs of the creation and annihilation operators. Thus the cubic term in the expansion of the Hamiltonian describes three-wave processes (the first term in $H_{1}$ is responsible for processes of decay of one wave into

\footnotetext{
${ }^{3}$ Here we assume that the nonlinear interaction is weak so that the energy sign of the nonlinear medium coincides with the sign of its quadratic Hamiltonian.
} 
three waves, the second corresponds to simultaneous creation of three waves), the next term describes four-wave processes, etc.

It is necessary to say that a calculation of matrix elements in this scheme assumes a pure algebraic procedure that consists in a substitution of the transformation (3.5) into the corresponding Hamiltonian, a forthcoming simplification and a symmetrization of the final result.

For a medium described by several pairs of canonical variables and when $H_{0}$ is diagonalized, several wave branches can appear, with dispersion laws $\omega_{i}(k)$ and amplitudes $a_{i}(k)$. In this case a summation over all types of waves in each term of the expansion is needed.

\section{Canonical Variables in Hydrodynamics}

As a first example we consider the equations of potential flow of an ideal compressible barotropic fluid, in which the pressure $p$ is a single-valued function of the density $\rho$. These equations can be written in the form:

$$
\begin{gathered}
\frac{\partial \rho}{\partial t}+\operatorname{div} \rho \nabla \varphi=0, \\
\frac{\partial \varphi}{\partial t}+\frac{(\nabla \varphi)^{2}}{2}+w(\rho)=0 .
\end{gathered}
$$

Here $\varphi$ is the velocity potential, $\omega(\rho)=\partial \varepsilon / \partial \rho$ is the enthalpy, where $\varepsilon(\rho)$ denotes the internal energy density. These equations conserve the energy

$$
H=\int\left[\frac{\rho(\nabla \varphi)^{2}}{2}+\varepsilon(\rho)\right] d \mathbf{r}
$$

It can be checked that the equation set (4.1), (4.2) can be represented in the form of the Hamilton equations:

$$
\frac{\partial \varphi}{\partial t}=\frac{\delta H}{\delta \rho}, \frac{\partial \rho}{\partial t}=-\frac{\delta H}{\delta \varphi} .
$$

Thus the density $\rho$ is a generalized coordinate, and $\varphi$ is the generalized momentum.

This result can also be obtained from a Lagrangian approach. In this case one makes use of the well-known expression for the Lagrangian of a mechanical system, generalized to the continuous case, and supplement it by the constraint:

$$
\frac{\partial \rho}{\partial t}+\operatorname{div} \rho \mathbf{v}=0
$$

Then the action is

$$
S=\int L d t=\int\left[\frac{\rho \mathbf{v}^{2}}{2}-\varepsilon(\rho)+\varphi\left(\frac{\partial \rho}{\partial t}+\operatorname{div} \rho \mathbf{v}\right)\right] d \mathbf{r} d t
$$


Its variation with respect to the variable $\mathbf{v}$ leads to the condition for the potential flow, $\mathbf{v}=\nabla \varphi$, and variations with respect to the variables $\rho$ and $\varphi$ lead to Eqs. (4.1), (4.2). Here the transition to the Hamiltonian is accomplished by the standard formula

$$
H=\int \varphi \frac{\partial \rho}{\partial t} d \mathbf{r}-L
$$

and leads us to (4.3).

We give the expression for the coefficients of the Hamiltonian expansion.

The diagonalization of

$$
H_{0}=\int\left[\frac{1}{2} \rho_{0}(\nabla \varphi)^{2}+c_{s}^{2} \frac{\delta \rho^{2}}{2 \rho_{0}}\right] d \mathbf{r}
$$

can be made by the transformation

$$
\begin{gathered}
\varphi_{k}=-\frac{i}{k}\left(\frac{\omega_{k}}{2 \rho_{0}}\right)^{1 / 2}\left(a_{k}-a_{-k}^{*}\right), \\
\delta \rho_{k}=k\left(\frac{\rho_{0}}{2 \omega_{k}}\right)^{1 / 2}\left(a_{k}+a_{-k}^{*}\right) .
\end{gathered}
$$

Here $\omega_{k}=k c_{s}$ refers to the eigen frequency, $\delta \rho=\rho-\rho_{0}$ is the deviation of the density from the equilibrium $\rho_{0}, c_{s}=\left(\partial p / \partial \rho_{0}\right)^{1 / 2}$ is the sound velocity. Substitution of this transformation in the next term of the expansion $H_{1}$,

$$
H_{1}=\int\left[\frac{1}{2} \delta \rho(\nabla \varphi)^{2}+c_{s}^{2} g \frac{\delta \rho^{3}}{2 \rho_{0}^{2}}\right] d \mathbf{r}
$$

gives the following expression for $U_{k k_{1} k_{2}}$ and $V_{k k_{1} k_{2}}$ :

$$
\begin{gathered}
U_{k k_{1} k_{2}}=V_{k k_{1} k_{2}} \\
=\frac{1}{16\left(\pi^{3} \rho_{0}\right)^{1 / 2}}\left\{3 g c_{s}^{2} \frac{k k_{1} k_{2}}{\left(\omega_{k} \omega_{k_{1}} \omega_{k_{2}}\right)^{1 / 2}}+\left(\frac{\omega_{k} \omega_{k_{1}}}{\omega_{k_{2}}}\right)^{1 / 2} k_{1} \frac{\left(\mathbf{k k}_{1}\right)}{k k_{1}}\right. \\
\left.+\left(\frac{\omega_{k} \omega_{k_{24}}}{\omega_{k_{1}}}\right)^{1 / 2} k_{1} \frac{\left(\mathbf{k k}_{2}\right)}{k k_{2}}+\left(\frac{\omega_{k_{2}} \omega_{k_{1}}}{\omega_{k}}\right)^{1 / 2} k \frac{\left(\mathbf{k}_{2} \mathbf{k}_{1}\right)}{k_{2} k_{1}}\right\} .
\end{gathered}
$$

The equations describing nonlinear sound waves in media with dispersion belong to the same type of system as (4.1), (4.2). These equations can be derived when considering the internal energy of the system $\varepsilon_{i n}$ as a functional of the density. This functional can be represented as a power series in $\nabla \rho$ :

$$
\mathcal{E}_{i n}=\int\left\{\varepsilon(\rho)+\frac{\nu}{2}(\nabla \rho)^{2}+\ldots\right\} d \mathbf{r} .
$$


Classical hydrodynamics corresponds to keeping only the first term in the above series. If we now include the second term, we get the Boussinesq system:

$$
\begin{gathered}
\frac{\partial \rho}{\partial t}+\operatorname{div} \rho \nabla \varphi=0 \\
\frac{\partial \varphi}{\partial t}+\frac{1}{2}(\nabla \varphi)^{2}=-\frac{\delta \mathcal{E}_{i n}}{\delta \rho}=-w(\rho)-\nu \triangle \rho .
\end{gathered}
$$

The Hamiltonian in this case coincides with the total energy of the system, i.e., with a sum of kinetic energy and internal energy given by (4.6), for while $\rho$ and $\varphi$ remain the canonical conjugated variables.

Introduction of canonical variables is possible also when we include vortex motion in an ideal fluid $[17,19,20]$. To this aim we must start from the full Euler equations of hydrodynamics:

$$
\begin{gathered}
\frac{\partial \rho}{\partial t}+\operatorname{div} \rho \mathbf{v}=0 \\
\frac{\partial \mathbf{v}}{\partial t}+(\mathbf{v} \nabla) \mathbf{v}=-\frac{\nabla p(\rho)}{\rho}=-\nabla w(\rho) .
\end{gathered}
$$

We know that, for the Euler equations in accordance with the Kelvin theorem, the circulation of the fluid velocity around any closed contour moving together with the fluid is conserved. In other words, in such a system there is a certain scalar function $\mu(\mathbf{r}, t)$ which is convected by the fluid and described by the following equation:

$$
\frac{d \mu}{d t}=\left(\frac{\partial}{\partial t}+\mathbf{v} \nabla\right) \mu=0
$$

Therefore, in formulating the variational principle we should include this equation as a constraint which implies to set

$$
L=\int\left[\frac{\rho \mathbf{v}^{2}}{2}-\varepsilon(\rho)+\varphi\left(\frac{\partial \rho}{\partial t}+\operatorname{div} \rho \mathbf{v}\right)-\lambda\left(\frac{\partial \mu}{\partial t}+\mathbf{v} \nabla \mu\right)\right] d \mathbf{r} .
$$

The variation of $L$ with respect to the variables $\mathbf{v}$ and $\mu$ leads to the following equations:

$$
\begin{gathered}
\mathbf{v}=\frac{\lambda}{\rho} \nabla \mu+\nabla \varphi \\
\frac{\partial \varphi}{\partial t}+(\mathbf{v} \nabla \varphi)-\frac{\mathbf{v}^{2}}{2}+w(\rho)=0 \\
\frac{\partial \lambda}{\partial t}+\operatorname{div} \lambda \mathbf{v}=0
\end{gathered}
$$

Here the first equation is the well-known change to the Clebsch variables $\lambda$ and $\mu$; the second one represents the generalization of the Bernoulli equation to the nonpotential 
flows and the last one governs the dynamics of a new variable $\lambda$. The choice of $\lambda$ and $\mu$ for a given value of $\mathbf{v}$ is not unique.

Let us consider two sets of potentials $\lambda, \mu, \varphi$, giving the same value for the velocity $\mathbf{v}$ with the help of Eq.(4.11). Multiplying (4.11) by the differential $d \mathbf{r}$ (for fixed time $t$ ), we get a relation between the new and old variables:

$$
d \varphi+\frac{\lambda}{\rho} d \mu=d \varphi^{\prime}+\frac{\lambda^{\prime}}{\rho} d \mu^{\prime}
$$

or

$$
d f \equiv d\left(\varphi-\varphi^{\prime}\right)=-\frac{\lambda}{\rho} d \mu-\frac{\lambda^{\prime}}{\rho} d \mu^{\prime}
$$

The last relation shows that $\varphi^{\prime}-\varphi$ is the generating function $f$ of a gauge transformation, depending on $\mu$ and $\mu^{\prime}$. The old and new canonical coordinates are then expressed in terms of the generating function by means of the formulas [21]

$$
\lambda=-\rho \frac{\partial f}{\partial \mu}, \lambda^{\prime}=\rho \frac{\partial f}{\partial \mu^{\prime}}
$$

determining the nonuniqueness in the choice of Clebsch variables.

Substituting the velocity $\mathbf{v}$ expressed in terms of the variables $\lambda, \mu$ and $\varphi$ directly into the Euler equation (4.8), we verify that

$$
\frac{\lambda}{\rho} \nabla\left(\frac{\partial \mu}{\partial t}+(\mathbf{v} \nabla) \mu\right)+\nabla \mu\left(\frac{\partial}{\partial t} \frac{\lambda}{\rho}+(\mathbf{v} \nabla) \frac{\lambda}{\rho}\right)+\nabla\left(\frac{\partial \varphi}{\partial t}+(\mathbf{v} \nabla) \varphi-\frac{\mathbf{v}^{2}}{2}+w(\rho)\right)=0 .
$$

Thus this equation is satisfied if Eq-s. (4.12), (4.13) are also imposed. If it is so the system of equations of hydrodynamics can be said to be equivalent to the system (4.7), (4.9), (4.12), (4.13). This is based on the uniqueness of the solution of the Cauchy problem for the original system and the one obtained (that is, rigorously speaking, an assumption). In doing this, we must in addition, by means of the velocity $\mathbf{v}$ given at the initial time, construct some set of functions $\lambda_{0}, \mu_{0}$ and $\varphi_{0}$, appearing as initial conditions for the system (4.9), (4.12), (4.13).

Now changing to a Hamiltonian description, we get:

$$
\begin{aligned}
& \frac{\partial \rho}{\partial t}=\frac{\delta H}{\delta \varphi}, \frac{\partial \varphi}{\partial t}=-\frac{\delta H}{\delta \rho} \\
& \frac{\partial \lambda}{\partial t}=\frac{\delta H}{\delta \mu}, \frac{\partial \mu}{\partial t}=-\frac{\delta H}{\delta \lambda}
\end{aligned}
$$

where the Hamiltonian

$$
H=\int\left[\frac{\rho \mathbf{v}^{2}}{2}+\varepsilon(\rho)\right] d \mathbf{r}
$$


coincides with the total energy of the system. For potential flows $(\lambda=\mu=0)$ we again arrive at a pair of canonical variables $(\rho, \varphi)$.

Canonical variables for the equations of relativistic hydrodynamics,

$$
\begin{gathered}
\frac{\partial \rho}{\partial t}+\operatorname{div}(\rho \mathbf{v})=0 \\
\left(\frac{\partial}{\partial t}+(\mathbf{v} \nabla)\right) \mathbf{p}+m \nabla w(\rho)=0 \\
\mathbf{p}=m \mathbf{v}\left(1-v^{2} / c^{2}\right)^{-1 / 2}
\end{gathered}
$$

are introduced in analogy to (4.11). In this case,

$$
\frac{\mathbf{p}}{m}=\frac{\lambda}{\rho} \nabla \mu+\nabla \varphi
$$

Just as in the preceding example, the variables $(\lambda, \mu)$ and $(\rho, \varphi)$ form pairs of canonically conjugate quantities, subjected to Eqs (4.12), with the Hamiltonian

$$
H=\int\left[\frac{\rho}{m}\left(m^{2} c+p^{2} c^{2}\right)^{1 / 2}+\varepsilon(\rho)\right] d \mathbf{r}
$$

A natural generalization of the Clebsch formulation (4.8) is the introduction of canonical variables for nonbarotropic flows [21], when $\varepsilon$ depends on the density as well as on the entropy $S$. For this the equations of motion (4.9), (4.11) are supplemented by the equation for the entropy advected by the fluid,

$$
\left(\frac{\partial}{\partial t}+(\mathbf{v} \nabla)\right) S=0
$$

and the thermodynamic relation

$$
d \varepsilon=\rho T d S+w d \rho,
$$

with $T$ as the temperature. In this case the transition to the new variables is accomplished by the formula

$$
\mathbf{v}=\nabla \varphi+\frac{\lambda}{\rho} \nabla \mu+\frac{\beta}{\rho} \nabla S
$$

For such flows $(\varphi, \rho, \lambda, \mu)$ and $(S, \beta)$ are pairs of canonically conjugate quantities:

$$
\begin{gathered}
\frac{\partial \rho}{\partial t}=\frac{\delta H}{\delta \varphi}=-\operatorname{div} \rho \mathbf{v} \\
\frac{\partial \varphi}{\partial t}=-\frac{\delta H}{\delta \rho}=\frac{\mathbf{v}^{2}}{2}-\mathbf{v} \nabla \varphi-w
\end{gathered}
$$




$$
\begin{gathered}
\frac{\partial \lambda}{\partial t}=\frac{\delta H}{\delta \mu}=-\operatorname{div} \lambda \mathbf{v}, \\
\frac{\partial \mu}{\partial t}=-\frac{\delta H}{\delta \lambda}=-\mathbf{v} \nabla \mu, \\
\frac{\partial \beta}{\partial t}=\frac{\delta H}{\delta S}=-\operatorname{div} \beta \mathbf{v}+\rho T, \\
\frac{\partial S}{\partial t}=-\frac{\delta H}{\delta \beta}
\end{gathered}
$$

where $H=\int\left[\rho\left(\mathbf{v}^{2} / 2\right)+\varepsilon(\rho, s)\right] d \mathbf{r}$. The equivalence of these equations to the equations of hydrodynamics is verified by direct substitution of the velocity in the Euler equation (4.8). Thus, in comparison with the barotropic case the number of canonical variables increases by two.

Now let us raise the natural question: What is the minimal number of the canonical conjugated pairs for describing any flow? As we saw above, introducing new canonical variables in the framework of the Lagrangian approach was connected with the addition of new constraints into the Lagrangian. For example, for the Lagrangian (4.10) they were the continuity equation for the density and the equation for the Lagrangian (material) invariant $\mu$ advected by the fluid. In the nonbarotropic case the new Lagrangian invariant, i.e, the entropy $S$, was added.

To describe the fluid in terms of the Lagrangian (material) variables it is enough to give three values $\left(a_{1}, a_{2}, a_{3}\right)=\mathbf{a}$ which, in the simplest case, coincide with the initial positions of each fluid particles, so that the coordinate of the particle at time $t$ will be equal to

$$
\mathbf{r}=\mathbf{r}(\mathbf{a}, t)
$$

Hence it becomes clear that originally there are three independent Lagrangian invariants,

$$
\mathbf{a}=\mathbf{a}(\mathbf{r}, t),
$$

that are the inverse map to (4.18). All other Lagrangian invariants are functions of a. If now we assign the equations for $\mathbf{a}$

$$
\frac{d \mathbf{a}}{d t} \equiv \frac{\partial \mathbf{a}}{\partial t}+(\mathbf{v} \nabla) \mathbf{a}=0
$$

as constraints ${ }^{4}$ in the Lagrangian for the fluid we immediately come to three new pairs of the canonical variables $\left\{\lambda_{l}, a_{l}\right\}(l=1,2,3)$ with the velocity in the form,

$$
\mathbf{v}=u_{l} \nabla a_{l} .
$$

Here $u_{l}=\lambda_{l} / \rho$ and the density $\rho$ is expressed through a my means of

$$
\rho=1 / J
$$

\footnotetext{
${ }^{4}$ These constraints are called often as the Lin's constraints [51]
} 
with $J=\operatorname{det}|| \hat{J}_{i j} \|$ as the Jacobian of the mapping (4.18), and $\hat{J}_{i j}=\partial x_{i} / \partial a_{j}$ being the Jacoby matrix (for more details, see two next sections). The vector $\mathbf{u}$ in this formula is expressed in terms of the velocity components $v_{i}$ by

$$
\mathbf{u}=\hat{J}^{t} \mathbf{v}
$$

where subscript $t$ means transpose.

The representation (4.19) is the most general one. In particular, all the changes of variables presented above follow from this formula. It can be simplified although remaining general.

Let us consider reversible smooth changes of variables

$$
\mathbf{a}=\mathbf{a}(\tilde{\mathbf{a}})
$$

Under these changes the representation (4.19) remains invariant,

$$
\mathbf{v}=\tilde{u}_{l} \nabla \tilde{a}_{l}
$$

but the vector $\mathbf{u}$ transforms as

$$
\tilde{u}_{l}=u_{k} \frac{\partial a_{k}}{\partial \tilde{a}_{l}} .
$$

If we now require that one of the component, say $u_{3}$, is equal to 1 , the representation (4.19) becomes (compare with [50])

$$
\mathbf{v}=\nabla \phi+\frac{\lambda_{1}}{\rho} \nabla \mu_{1}+\frac{\lambda_{2}}{\rho} \nabla \mu_{2}
$$

If now in this equation we put for $\mu_{2}$ the entropy $S$, then we come back to the transformation (4.17). Note that such a reduction is possible if the surface family of the constant entropy $S(\mathbf{r}, t)=$ const are homotopic to the one of the constant surfaces, related to, e.g., $a_{1}(\mathbf{r}, t)=$ const. Hence, in particular, it follows that in the barotropic case it is enough to take two pairs of the Clebsch variables in order to describe any fluid flow. One pair of the Clebsch variables, as we will see further, describes a partial type of flows. Nevertheless, locally any flow can be paratmerized by one pair of the Clebsch variables [17].

\section{$5 \quad$ Noncanonical Poisson Brackets}

Now let us consider how one introduces a Hamiltonian structure into hydrodynamics in terms of the natural physical variables. To do so, it is sufficient to construct a Poisson bracket that satisfies all the necessary requirements. The simplest way of constructing such brackets is to convert the Poisson bracket expressed in terms of canonical variables to a bracket in terms of the natural variables. Note that in this 
case the arisen symplectic operator occurs locally in those variables. As an example we carry out the conversion of the formula for barotropic flows of an ideal fluid. The calculations for other models can be done in exactly the same way.

According to (4.16), the Poisson bracket is given by the expression:

$$
\{F, G\}=\int\left\{\left[\frac{\delta F}{\delta \rho} \frac{\delta G}{\delta \varphi}-\frac{\delta F}{\delta \varphi} \frac{\delta G}{\delta \rho}\right]+\left[\frac{\delta F}{\delta \lambda} \frac{\delta G}{\delta \mu}-\frac{\delta F}{\delta \mu} \frac{\delta G}{\delta \lambda}\right]\right\} d \mathbf{r} .
$$

Here the velocity is expressed in terms of $\lambda, \mu$ and $\rho, \varphi$ by the formula

$$
\mathbf{v}=\nabla \varphi+\frac{\lambda}{\rho} \nabla \mu
$$

by means of which one can calculate the variational derivatives of $F$ with respect to $\rho, \varphi, \lambda$ and $\mu$ :

$$
\begin{gathered}
\left.\frac{\delta F}{\delta \rho}\right|_{\lambda}=\left.\frac{\delta F}{\delta \rho}\right|_{v}-\frac{\lambda \nabla \mu}{\rho} \frac{\delta F}{\delta \mathbf{v}}, \quad \frac{\delta F}{\delta \varphi}=-\operatorname{div} \frac{\delta F}{\delta \mathbf{v}}, \\
\frac{\delta F}{\delta \lambda}=\frac{\nabla \mu}{\rho} \frac{\delta F}{\delta \mathbf{v}}, \quad \frac{\delta F}{\delta \mu}=-\operatorname{div}\left(\frac{\lambda}{\rho} \frac{\delta F}{\delta \mathbf{v}}\right) .
\end{gathered}
$$

In these formulas the variational derivatives on the left of the equality signs are taken with fixed $\lambda, \mu, \rho, \varphi$, and those on the right for constant $\rho$ and $\mathbf{v}$. Substitution of these relations in (5.1) leads us to the bracket [15]

$$
\begin{aligned}
\{F, G\}= & \int\left\{\left(\nabla \frac{\delta F}{\delta \rho}, \frac{\delta G}{\delta \mathbf{v}}\right)-\left(\nabla \frac{\delta G}{\delta \rho}, \frac{\delta F}{\delta \mathbf{v}}\right)\right\} d \mathbf{r} \\
& +\int\left(\frac{\operatorname{curl} \mathbf{v}}{\rho},\left[\frac{\delta F}{\delta \mathbf{v}} \times \frac{\delta G}{\delta \mathbf{v}}\right]\right) d \mathbf{r}
\end{aligned}
$$

the Jacobi identity (2.9) being satisfied automatically.

In terms of this bracket, the continuity and Euler equations have the form

$$
\begin{gathered}
\frac{\partial \rho}{\partial t}=-\operatorname{div} \rho \mathbf{v}=\{\rho, H\} \\
\frac{\partial \mathbf{v}}{\partial t}=-(\mathbf{v}, \nabla) \mathbf{v}-\nabla w(\rho)=\{\mathbf{v}, H\}
\end{gathered}
$$

where $H=\int\left[\rho \mathbf{v}^{2} / 2+\varepsilon(\rho)\right] d \mathbf{r}$.

The bracket (5.3) has a more obvious meaning if we go over to the new variable $\mathbf{p}=\rho \mathbf{v}$, the momentum density. In these variables this bracket is changed to the Berezin-Kirillov-Kostant (BKK) bracket [14]:

$$
\{F, G\}=\int \rho\left\{\left(\nabla \frac{\delta F}{\delta \rho}, \frac{\delta G}{\delta \mathbf{p}}\right)-\left(\nabla \frac{\delta G}{\delta \rho}, \frac{\delta F}{\delta \mathbf{p}}\right)\right\} d \mathbf{r}
$$




$$
+\int\left(\mathbf{p},\left[\left(\frac{\delta G}{\delta \mathbf{p}} \nabla\right) \frac{\delta F}{\delta \mathbf{p}}-\left(\frac{\delta F}{\delta \mathbf{p}} \nabla\right) \frac{\delta G}{\delta \mathbf{p}}\right]\right) d \mathbf{r} .
$$

Using (5.4) to calculate brackets between components of $\mathbf{p}$ and $\rho$, we find that

$$
\begin{gathered}
\left\{p_{i}(\mathbf{r}), p_{j}\left(\mathbf{r}^{\prime}\right)\right\}=\left(p_{j}\left(\mathbf{r}^{\prime}\right) \nabla_{i}^{\prime}-p_{i}(\mathbf{r}) \nabla_{j}\right) \delta\left(\mathbf{r}-\mathbf{r}^{\prime}\right), \\
\left\{p_{i}(\mathbf{r}), \rho\left(\mathbf{r}^{\prime}\right)\right\}=\rho \nabla_{i} \delta\left(\mathbf{r}-\mathbf{r}^{\prime}\right) .
\end{gathered}
$$

In accordance with (2.12), these relations give a Lie algebra, which coincides with the algebra of vector fields $[60,14]$ in this case.

The brackets $(5.4),(5.5)$ can also be obtained in other ways. The simplest method is to regard the Poisson bracket as the classical limit of the corresponding quantum commutators, which were first calculated for hydrodynamics by L.D.Landau [12]. Another method for calculating the Poisson brackets for hydrodynamic models, proposed by G.E.Volovik and I.E.Dzyaloshinskii [13], is based on the fact that $\mathbf{p}$ and $\rho$ are the densities of the generators of translations and gauge transformations.

In a sake of completeness we give the expressions for the Poisson brackets for the hydrodynamic equations of ideal fluids for arbitrary dependence of the pressure on both the density and the entropy [15]

$$
\begin{gathered}
\{F, G\}=\int\left\{\left(\nabla \frac{\delta F}{\delta \rho}, \frac{\delta G}{\delta \mathbf{v}}\right)-\left(\nabla \frac{\delta G}{\delta \rho}, \frac{\delta F}{\delta \mathbf{v}}\right)\right\} d \mathbf{r} \\
+\int\left(\frac{\operatorname{curl} \mathbf{v}}{\rho},\left[\frac{\delta F}{\delta \mathbf{v}} \times \frac{\delta G}{\delta \mathbf{v}}\right]\right) d \mathbf{r}+\int\left(\frac{\nabla S}{\rho},\left[\frac{\delta F}{\delta \mathbf{v}} \frac{\delta G}{\delta S}-\frac{\delta G}{\delta \mathbf{v}} \frac{\delta F}{\delta S}\right]\right) d \mathbf{r} .
\end{gathered}
$$

We want to repeat once more that the introduction of the Poisson brackets to a system means that such systems possess the Hamiltonian structure in the weakest sense. For example, for the above equations of ideal hydrodynamics it reflects in the fact that the brackets expressed in terms of natural variables are degenerate, i.e, there exist annulators of these Poisson brackets (Casimirs) which, as we will see in the next sections, are connected with a specific gauge symmetry of the hydrodynamic equations, providing, in particular, the conservation of the fluid velocity circulation. Besides, it means that a direct conversion, as, i.e., passing from (5.3) or to (5.6) to the canonical basis is impossible in general. For this case at first we need to resolve all our constraints (Casimirs). A typical example just consists in introducing Clebsch variables. This is all the more interesting as, so far, we have not explicitly known how these Casimirs look like.

Of particular interest is the introduction of a Hamiltonian structure for the incompressible fluid. In this case $\rho$ is no longer an independent variable, and can be eliminated by using the relation $\operatorname{div} \mathbf{v}=0$. Thus in the limit of the incompressible fluid there is only one pair of canonical variables $\lambda$ and $\mu$, and the Poisson bracket in this case takes the form

$$
\{F, G\}=\int\left[\frac{\delta F}{\delta \lambda} \frac{\delta G}{\delta \mu}-\frac{\delta F}{\delta \mu} \frac{\delta G}{\delta \lambda}\right] d \mathbf{r} .
$$


By means of relations analogous to (5.2), one can derive

$$
\begin{gathered}
\frac{\delta F}{\delta \lambda}=\left(\frac{\nabla \mu}{\rho}, \frac{\delta F}{\delta \mathbf{v}}-\nabla \frac{1}{\Delta} \operatorname{div} \frac{\delta F}{\delta \mathbf{v}}\right), \\
\frac{\delta F}{\delta \mu}=-\operatorname{div} \frac{\lambda}{\rho}\left(\frac{\delta F}{\delta \mathbf{v}}-\nabla \frac{1}{\Delta} \operatorname{div} \frac{\delta F}{\delta \mathbf{v}}\right) .
\end{gathered}
$$

As a result, we arrive at the equation

$$
\{F, G\}=\int\left(\operatorname{curl} \mathbf{v},\left[\left(\frac{\delta F}{\delta \mathbf{v}}-\nabla \frac{1}{\Delta} \operatorname{div} \frac{\delta F}{\delta \mathbf{v}}\right) \times\left(\frac{\delta G}{\delta \mathbf{v}}-\nabla \frac{1}{\Delta} \operatorname{div} \frac{\delta G}{\delta \mathbf{v}}\right)\right]\right) d \mathbf{r} .
$$

(Here we put $\rho=1$.) This expression shows that the manifold $G$ coincides with the algebra of vector fields $\mathbf{A}(\mathbf{r})$ for which $\operatorname{div} \mathbf{A}=0$. This bracket is expressed in a more compact form using $\boldsymbol{\Omega}=$ curlv [18], to read

$$
\{F, G\}=\int\left(\Omega\left[\operatorname{curl} \frac{\delta F}{\delta \Omega} \times \operatorname{curl} \frac{\delta G}{\delta \Omega}\right]\right) .
$$

As a result, the Euler equation for $\boldsymbol{\Omega}$,

$$
\frac{\partial \boldsymbol{\Omega}}{\partial t}=\operatorname{curl}[\mathbf{v} \times \boldsymbol{\Omega}]
$$

becomes the Hamiltonian one $[9,18]$ :

$$
\frac{\partial \Omega}{\partial t}=\{\Omega, H\}
$$

where

$$
H=\int \frac{\mathbf{v}^{2}}{2} d \mathbf{r}
$$

The bracket (5.8) also gives a Hamiltonian structure for two-dimensional hydrodynamics. In this case $\Omega$ has a single component, which is conveniently expressed in terms of the stream function $\psi$ :

$$
\Omega=-\Delta \psi\left(v_{x}=\frac{\partial \psi}{\partial y}, v_{y}=-\frac{\partial \psi}{\partial x}\right)
$$

In the two-dimensional case the equation of motion (5.9) and the Poisson bracket (5.8) have the form:

$$
\frac{\partial \Omega}{\partial t}=\{\Omega, H\}=-\frac{\partial \Omega}{\partial x} \frac{\partial \psi}{\partial y}+\frac{\partial \Omega}{\partial y} \frac{\partial \psi}{\partial x} \equiv-\frac{\partial(\Omega, \psi)}{\partial(x, y)},
$$




$$
\begin{gathered}
\{F, G\}=\int \Omega \frac{\partial(\delta F / \delta \Omega, \delta G / \delta \Omega)}{\partial(x, y)} d x d y, \\
H=\frac{1}{2} \int(\nabla \psi)^{2} d x d y .
\end{gathered}
$$

A Hamiltonian structure is introduced analogously into the Rossby equation, which differs from (5.8) in having the additional term $\beta(\partial \psi / \partial x)$ entering [1]:

$$
\frac{\partial}{\partial t} \Delta \psi+\beta \frac{\partial \psi}{\partial x}=-\frac{\partial(\Delta \psi, \psi)}{\partial(x, y)} .
$$

It is then easy to see that the change $\Omega \rightarrow \Omega-\beta y$ reduces this equation to (5.10). Thus, the Poisson bracket for (5.11) is given analogously by

$$
\{F, G\}=\int(\Omega+\beta y) \frac{\partial(\delta F / \delta \Omega, \delta G / \delta \Omega)}{\partial(x, y)} d x d y
$$

while the Hamiltonian $H$ is still defined by the earlier expression

$$
H=\frac{1}{2} \int(\nabla \psi)^{2} d x d y
$$

To the said above one should add that the Poisson brackets (5.10) and (5.12) for flows with closed stream lines can be reduced to the Gardner-Zakharov-Faddeev brackets used in the theory of integrable equations [40]. Details of such consideration can be found in the original papers [38, 39].

Thus, introducing noncanonical Poisson brackets on the base of canonical ones represents the most simple way of their finding. Moreover the Hamiltonian structure given by means of these brackets is the weakest Hamiltonian formulation of the equations. In this formulation, in particular, it is impossible to write explicitly the variational principle. From another side, as it will be shown later, the representation of the hydrodynamic type equations by means of the noncanonical Poisson bracket can be written for arbitrary flows. However, for the arbitrariness one should pay by the bracket degeneracy, i.e., by existence of Casimirs annulling noncanonical brackets.

\section{Ertel's Theorem}

In this section and in the next ones we show, by following mainly results expounded in $[41,43]$, that for perfect fluids with arbitrary dependence of the pressure on the fluid density and entropy the Ertel's theorem as well as the Kelvin theorem about the conservation of the velocity circulation are a consequence of the specific gauge symmetry connected with the relabeling of fluid particles. We discuss also the role played by this symmetry in the Hamiltonian structures. 
The Ertel's theorem [42] for a perfect fluid says that the quantity

$$
I_{L}=\frac{(\Omega \nabla S)}{\rho}
$$

is the Lagrangian invariant. Here $\Omega=$ curlv is the vorticity, $\mathbf{v}$ is the fluid velocity which satisfies the Euler equation,

$$
\frac{\partial \mathbf{v}}{\partial t}-(\mathbf{v} \nabla) \mathbf{v}=-\frac{\nabla p}{\rho}
$$

and $S$ the specific entropy advected by the fluid,

$$
\frac{\partial S}{\partial t}+(\mathbf{v} \nabla) S=0
$$

The density $\rho$ is defined from the continuity equation,

$$
\frac{\partial \rho}{\partial t}+\operatorname{div}(\rho \mathbf{v})=0
$$

We omit a proof of this theorem validity of which can be checked by direct calculations (see, for instance, [53]).

The invariance of $I_{L}$ means that $I_{L}$ depends only on the Lagrangian coordinates a, and it does not change in time moving together with a fluid particle.

As was mentioned before, the choice of the Lagrangian variables is arbitrary: they label each fluid particle. Therefore often these coordinates are called as the Lagrangian markers. Usually the Lagrangian coordinates are chosen to coincide with the initial positions of fluid particles, $\left.\mathbf{r}\right|_{\mathbf{t}=\mathbf{0}}=\mathbf{a}$. Thus, a transition from one (Euler) description to another (Lagrangian) one is accomplished by means of the change of variables

$$
\mathbf{r}=\mathbf{r}(\mathbf{a}, t)
$$

with a being a label of each fluid particle. Velocity of the particle at the point $\mathbf{r}$ is given by the usual formula

$$
\mathbf{v}(\mathbf{r}, \mathbf{t})=\left.\dot{\mathbf{r}}\right|_{\mathbf{a}}
$$

where dot means derivative with respect to time $t$. In terms of the Lagrangian variables, the solution to the equations (6.4) and (6.3) can be written as follows,

$$
\rho(\mathbf{r}, t)=\rho_{0}(\mathbf{a}) / J, \quad S(\mathbf{r}, t)=S_{0}(\mathbf{a})
$$

where $J=\operatorname{det}\left\|\hat{J}_{i j}\right\|$ is a Jacobian and

$$
\hat{J}_{i \alpha}=\frac{\partial x_{i}}{\partial a_{\alpha}}
$$


is a Jacobi matrix of the mapping (6.5), which is assumed to be one-to-one. Further we will suppose $J \neq 0$ everywhere, that guarantee the existence of the mapping inverse to (6.5). The Jacobi matrix plays the basic role. Its knowledge allows to determine not only the main flow parameters but also its geometrical characteristics, in particular the metric tensor. The equation of motion for the Jacobi matrix follows directly from the definition of the velocity (6.6). Consider the vector $\delta \mathbf{r}$ connected two nearest fluid particles,

$$
\delta \mathbf{r}=\mathbf{r}(\mathbf{a}+\delta \mathbf{a}, \mathbf{t})-\mathbf{r}(\mathbf{a}, \mathbf{t}) .
$$

Using the definition (6.6) it is easy to get the equation for this quantity,

$$
\frac{d \delta \mathbf{r}}{d t}=(\delta \mathbf{r}, \nabla) \mathbf{v}
$$

Expanding then $\delta \mathbf{r}$ relative to the small vector $\delta \mathbf{a}$,

$$
\delta x_{i}=\hat{J}_{i j} \delta_{j}
$$

we arrive at the equation of motion for the Jacobi matrix,

$$
\frac{d}{d t} \hat{J}=U \hat{J}
$$

containing the matrix elements

$$
U_{i j}=\frac{\partial v_{i}}{\partial x_{j}}
$$

The symmetric part of $U$,

$$
B=\frac{1}{2}\left(U+U^{T}\right)
$$

is a stress tensor, and its antisymmetric part corresponds to the vorticity,

$$
\Omega=\frac{1}{2}\left(U-U^{T}\right)
$$

Hence the equation for the matrix inverse to $\hat{J}$ is

$$
\frac{d}{d t} \hat{J}^{-1}=-\hat{J}^{-1} U
$$

that in the component notation has the form

$$
\frac{d}{d t} \frac{\partial a_{\alpha}}{\partial x_{i}}=-\frac{\partial a_{\alpha}}{\partial x_{j}} \frac{\partial v_{j}}{\partial x_{i}} .
$$

The metric tensor is defined by means of distances between two nearest Lagrangian particles,

$$
\left(\delta x_{i}\right)^{2}=g_{i k} \delta a_{i} \delta a_{k},
$$


and equal to

$$
g_{i k}=\hat{J}_{l i} \hat{J}_{l k}
$$

The invariant $I_{L}$ is local in Lagrangian variables. Therefore if one takes its convolution with arbitrary function $f(\mathbf{a})$, then one can get the infinite family of the conservation laws in the integral form

$$
I_{i}=\int I_{L}(\mathbf{a}) f(\mathbf{a}) d \mathbf{a} .
$$

To begin with, we show that for barotropic fluids (when pressure $p$ depends only on the density $\rho$ ) the Kelvin theorem follows from this relation. Notice that in this case there is one additional freedom: the entropy $S$ has no link with the pressure and therefore instead of $S$ in (6.2) and (6.7) we can take an arbitrary function of Lagrangian markers a. Also one should note that in the first equation of (6.7), without any loose of generality, one can set $\rho_{0}(\mathbf{a})=1^{5}$, so that

$$
\rho(\mathbf{r}, t)=1 / J .
$$

Substitute (6.1) into (6.13) and integrate once by parts. With account of (6.7) and $J d \mathbf{a}=d \mathbf{r}$, we get

$$
I_{i}=\int(\mathbf{v},[\nabla f \times \nabla S]) d \mathbf{r} .
$$

Here the gradient is taken with respect to $\mathbf{r}$, but functions $f$ and $S_{0}$ are functions of $\mathbf{a}=\mathbf{a}(\mathbf{x}, t)$. Therefore come back again to the integration against $\mathbf{a}$. As a result of simple algebra we arrive at the expression,

$$
I_{i}=\int \dot{x}_{i} J \epsilon_{i j k} \frac{\partial a_{\alpha}}{\partial x_{j}} \frac{\partial a_{\beta}}{\partial x_{k}} \frac{\partial f(a)}{\partial a_{\alpha}} \frac{\partial S_{0}(a)}{\partial a_{\beta}} d \mathbf{a} .
$$

Taking then into account the identity

$$
J \epsilon_{i j k} \frac{\partial a_{\alpha}}{\partial x_{j}} \frac{\partial a_{\beta}}{\partial x_{k}}=\epsilon_{\alpha \beta \gamma} \frac{\partial x_{i}}{\partial a_{\gamma}}
$$

the integral is transformed into

$$
I_{i}=\int A_{j}(\mathbf{a}) \dot{x}_{i} \frac{\partial x_{i}}{\partial a_{j}} d \mathbf{a}
$$

Here the vector function $\mathbf{A}(\mathbf{a})$ reads:

$$
\mathbf{A}(\mathbf{a})=\left[\nabla f \times \nabla S_{0}\right] .
$$

It has a zero divergence:

$$
\operatorname{div} \mathbf{A}(\mathbf{a})=0 .
$$

\footnotetext{
${ }^{5}$ It corresponds to such change of variables $\mathbf{b}=\mathbf{b}(\mathbf{a})$ which eliminates $\rho_{0}: J_{a b}=\rho_{0}$
} 
Note that till now we have never used the fact that the fluid is barotropic, i.e., the equation (6.17) is applicable for any equation of state including the general dependence of the pressure on both the density and the entropy. For the barotropic case the entropy $S_{0}$ can be considered as an arbitrary function of $\mathbf{a}$. Therefore $A(a)$ can be considered also as arbitrary with the only constraint (6.19).

Let this vector function $\mathbf{A}(\mathbf{a})$ be concentrated on some closed curve: it is equal to zero everywhere outside this curve. We will parameterize the curve by the arc length $s$,

$$
\mathbf{a}=\mathbf{a}(s) \text { with } \mathbf{a}(s+l)=\mathbf{a}(\mathbf{s})
$$

where $l$ is the curve length.

It is then easy to check that the function

$$
\mathbf{A}=\int_{0}^{l} \frac{d \mathbf{a}(s)}{d s} \delta(\mathbf{a}-\mathbf{a}(s)) d s
$$

satisfies all necessary conditions: it concentrates on the curve $\mathbf{a}=\mathbf{a}(s)$ and has zero divergence. Plugging this formula into the integral (6.17), after simple integration, we come to the Kelvin theorem for the barotropic fluid:

$$
I_{K}=\int_{C}(\mathbf{v}(\mathbf{r}, \mathbf{t}), d \mathbf{l})
$$

Here the contour $C$, moving together with the fluid, is the image of the closed curve (6.20). Thus, we have shown that the Kelvin's theorem is a direct consequence of the Ertel's theorem applied to the case of barotropic fluids.

The Kelvin theorem is valid also for arbitrary dependence $p(\rho, S)$. This property is not widely known in the literature, for instance, it is absent in the Landau-Lifshits course [53]. Curiously, the answer in this case will have the same form as (6.21). The only difference will be connected with a choice of contour. For the barotropic case, as we saw before, the only restriction was connected with the condition (6.19) which provides the closure of the contour. For the general dependence $p=p(\rho, S)$ in addition to (6.19) one needs to satisfy the condition (6.18). According to the latter the lines of the vector field $\mathbf{A}$ must lie on the surfaces of the constant entropy $S_{0}(\mathbf{a})$. Therefore if we choose the closed contour lying on this (fluid!) surface we immediately arrive at the Kelvin theorem (6.21). Thus, the Kelvin theorem in the general case says that the velocity circulation is conserved in time if the fluid contour lies on the surface $S(\mathbf{a}(\mathbf{r}, t))=$ const advected by the fluid.

To the end of this section we pay attention to one interesting interpretation of the Kelvin theorem. According to [11] conservation of the velocity circulation can been considered as a sequence of conservation of the relative Poincare invariant

$$
\oint \mathbf{p} d \mathbf{q}
$$


For barotropic flows to each fluid particle one can correspond the Hamiltonian

$$
h=\frac{p^{2}}{2}+w(\rho)
$$

where $\mathbf{p}=\dot{\mathbf{r}}$, and the enthalpy $w$ plays a role of its potential energy.

If now one takes instead of the contour in (6.22) the fluid one then it is seen that the Poincare invariant will coincide with the velocity circulation

$$
\oint \mathbf{v} d \mathbf{r}
$$

and, thus, the Kelvin theorem becomes a direct consequence of a conservation of the relative Poincare invariant.

This concept has been occurred to be very useful for other hydrodynamic systems, in particular, for some problems in plasma physics $[58,57]$, when the motion of a fluid particle can be reduced to the Hamilton equation for a charge particle in a magnetic field in a presence of a self-consistent potential. In such cases the analog of the Kelvin theorem is simply a sequence of conservation of the relative Poincare invariant.

\section{Gauge Symmetry - Relabeling Group}

In this section we consider how the conservation of the Ertel invariants follows from the variational principle.

To begin, we make two remarks.

Firstly, let $\mathbf{I}_{\mathbf{l}}=\left(I_{1}, \ldots, I_{n}\right)$ be a set of Lagrangian invariants, each of them moving with the fluid and respectively

$$
\frac{d I_{k}}{d t}=\frac{\partial I_{k}}{\partial t}+\mathbf{v} \nabla I_{k}=0
$$

Then any function of $\mathbf{I}_{\mathbf{l}}$ will also be a Lagrangian invariant. To construct an Eulerian conservative density from the given Lagrangian one it is enough to be convinced that the quantity $I_{e u}=\rho I_{k}$ obeys the continuity equation

$$
\frac{\partial I_{e u}}{\partial t}+\operatorname{div}\left(I_{e u} \mathbf{v}\right)=0
$$

The equations of ideal hydrodynamics, as we saw above, have two Lagrangian invariants, i.e., the Ertel invariant $I_{L}(6.1)$ and $s^{6}$. Both these integrals generate the following conservation law

$$
I_{i}=\int \rho f\left(I_{L}, s\right) d \mathbf{r}
$$

\footnotetext{
${ }^{6}$ To avoid a confusion only in this section we denote the entropy as $s$, everywhere outside this section the entropy remains the previous notation $S$.
} 
with $f\left(I_{L}, s\right)$ being an arbitrary function of its arguments.

Secondly, the Euler equation (6.2) in terms of the Lagrangian variables is nothing else but the Newton equation for a fluid particle,

$$
\ddot{x}_{i}=-\frac{\nabla_{i} p}{\rho} .
$$

Acting by the Jacobi matrix $\hat{J}$ to the both sides of this equation gives

$$
\frac{\partial x_{i}}{\partial a_{k}} \ddot{x}_{i}=-\frac{1}{\rho} \frac{\partial p(\rho, s)}{\partial a_{k}} .
$$

This equation in the form (7.2) or (7.3) is closed by means of Eqs. (6.7) and (6.14).

The action in terms of the Lagrangian (material) variables is written in the same form as in classical mechanics,

$$
S=\int d t L=\int d t d \mathbf{r}\left(\rho \frac{\dot{x}_{i}^{2}}{2}-\varepsilon(\rho, s)\right)
$$

where $\varepsilon$ is the internal energy density connected with the enthalpy $w$ by means of the thermodynamic relation,

$$
d \varepsilon=\rho T d s+w d \rho
$$

with $T$ as the temperature.

Let us now check that varying the action, $\delta S=0$, is equivalent to the equation of motion (7.3).

At first let us pass in (7.4) from integration over $\mathbf{r}$ to $\mathbf{a}$. As a result, the action can then be transformed as follows,

$$
S=\int d t d \mathbf{a}\left(\frac{\dot{x}_{i}^{2}}{2}-\tilde{\varepsilon}(\rho, s)\right) .
$$

Here the time derivative of $x$ is taken for fixed $a, \tilde{\varepsilon}=\varepsilon / \rho$ is the function of $\rho$ and $s$ which are defined with the help of relations (6.7), (6.14). Because only $\rho$ in the internal energy $\tilde{\varepsilon}$ contains the dependence of $x$ through the Jacobian (6.14), the main difficulty with a variation will be connected with the second term in (7.6).

Using both the identity (6.16) and the formula

$$
J=\frac{1}{6} \epsilon_{i j k} \epsilon_{\alpha \beta \gamma} \frac{\partial x_{i}}{\partial a_{\alpha}} \frac{\partial x_{j}}{\partial a_{\beta}} \frac{\partial x_{k}}{\partial a_{\gamma}}
$$

one can get

$$
\begin{gathered}
\delta S=\int d t d \mathbf{a}\left(-\ddot{x}_{i} \delta x_{i}+\rho^{2} \frac{\partial \tilde{\varepsilon}}{\partial \rho} \delta J\right) \\
=\int d t d \mathbf{a}\left(-\ddot{x}_{i}-\frac{1}{2} \frac{\partial}{\partial a_{\alpha}}\left(\rho^{2} \frac{\partial \tilde{\varepsilon}}{\partial \rho}\right) \epsilon_{\alpha \beta \gamma} \epsilon_{i j k} \frac{\partial x_{j}}{\partial a_{\beta}} \frac{\partial x_{k}}{\partial a_{\gamma}}\right) \delta x_{i}
\end{gathered}
$$




$$
=\int d t d \mathbf{a}\left(-\ddot{x}_{i}-\frac{1}{\rho} \frac{\partial}{\partial a_{\alpha}}\left(\rho^{2} \frac{\partial \tilde{\varepsilon}}{\partial \rho}\right) \frac{\partial a_{\alpha}}{\partial x_{i}}\right) \delta x_{i}=0
$$

or

$$
\ddot{x}_{i}=-\frac{1}{\rho} \frac{\partial}{\partial a_{\alpha}}\left(\rho^{2} \frac{\partial \tilde{\varepsilon}}{\partial \rho}\right) \frac{\partial a_{\alpha}}{\partial x_{i}} .
$$

Hence it is seen that the resulting equation coincides with the equation of motion (7.3) if one puts

$$
p(\rho, s)=\rho^{2} \frac{\partial \tilde{\varepsilon}}{\partial \rho} .
$$

(The last equality is a direct sequence of the thermodynamic relation (7.5).)

Thus, we have proved that the equations of motion of ideal fluid in the Lagrangian form follow directly from the variational principle.

The simplest conservation laws, i.e., the conservation of momentum

$$
\mathbf{P}=\int \dot{\mathbf{x}} d \mathbf{a}=\int \rho \mathbf{v}(\mathbf{r}, t) d \mathbf{r}
$$

and the conservation of energy,

$$
E=\int\left(\frac{\dot{\mathbf{x}}^{2}}{2}+\tilde{\varepsilon}(\rho, S)\right) d \mathbf{a}=\int\left(\frac{\rho v^{2}}{2}+\varepsilon(\rho, S)\right) d \mathbf{r}
$$

follow as a result of invariance of the action relative to two independent symmetries, translations in space and time.

The equations of hydrodynamics, as it was shown at first in [41], have an additional nontrivial symmetry connected with arbitrariness in the possible choices of the Lagrangian markers. Nothing has to depend on this choice: the fluid dynamics as well as the equations of motion remain the same. From all possible relabeling transformations, the action invariance requirement restrains some certain class. In the case of the barotropic fluids the action appears to be invariant if transformations $\mathbf{b}=\mathbf{b}(\mathbf{a})$ are incompressible, i.e., for which the Jacobian equal to 1 :

$$
J=\operatorname{det}\left\|\partial b_{i} / \partial a_{j}\right\|=1
$$

All these transformations form the group of diffeomorphisms preserving the volume. (It is interesting to note that the same group governs the motion of an incompressible fluid.) This symmetry, in accordance with the Noether theorem, generates new conservation laws. To find them it is enough to consider infinitesimal transformations. In the given case those are defined by

$$
\mathbf{b}=\mathbf{a}+\delta \mathbf{a}
$$

where the function $\delta \mathbf{a}=\alpha(\mathbf{a})$ satisfies the condition 


$$
\frac{\partial \alpha_{i}(\mathbf{a})}{\partial a_{i}}=0
$$

which is a direct sequence of Eq. (7.8).

For the general equation of state $p=p(\rho, s)$ the invariance of the action implies that the transformations should keep the surface $s=s(\mathbf{a})$ to remain, being simultaneously incompressible. As a result, on the function $\alpha(\mathbf{a})$ we have one additional constraint

$$
[\nabla s \times \alpha]=0 .
$$

If in the first case Eq.(7.9) can be resolved by introducing the vector potential

$$
\alpha=\operatorname{curl} \zeta
$$

for example, with the Coulomb gauge $\operatorname{div} \zeta=0$, then in the general case both equations (7.9) and (7.10) are satisfied if one puts

$$
\alpha=[\nabla s \times \nabla \psi]
$$

Here $\psi$ is a scalar function and gradient is taken with respect to a.

Omitting all the intermediate derivation of the conservation law (it is the standard procedure, for reference see, for instance, [83]) we present only the final answers:

i) For the barotropic fluid the conservation law has the form

$$
\frac{d}{d t}\left[\nabla_{a} \dot{x}_{i} \times \nabla_{a} x_{i}\right]=0
$$

or it gives the whole conserved vector

$$
\mathbf{I}_{\mathbf{L}}=\left[\nabla_{a} \dot{x}_{i} \times \nabla_{a} x_{i}\right] .
$$

This integral was known since the last century: it was found by Cauchy [17] (see also $[45,44])$.

Matrix notation of the equation (7.11) has the form:

$$
\hat{J} \frac{d \hat{J}^{T}}{d t}-\hat{J}^{T} \frac{d \hat{J}}{d t}=\Omega^{(0)},
$$

where index $T$ means transposition, and the matrix $\Omega^{(0)}$ is expressed through the vector invariant $\mathbf{I}_{\mathbf{L}}$ with the help of formula

$$
\Omega_{i j}^{(0)}=\epsilon_{i j k} I_{L k} .
$$

Recently this matrix representation of the equation (7.11) was used by the authors of the paper [59] to construct a set of exact three-dimensional solutions for the Euler equation for incompressible fluids. 
Returning to the Euler description and using the identity (6.16) this vector integral can be transformed into the form

$$
\mathbf{I}_{\mathbf{L}}=J(\boldsymbol{\Omega}, \nabla) \mathbf{a} \equiv \frac{\rho_{0}(\mathbf{a})}{\rho}(\boldsymbol{\Omega}, \nabla) \mathbf{a} .
$$

Here $\mathbf{a}$ is considered as a function of $\mathbf{r}$ and $t$. If $\mathbf{a}$ are the initial coordinates of fluid particles, then the vector $(7.12)$ can be expressed through the initial distributions $\Omega_{\mathbf{0}}(\mathbf{a})$ and $\rho_{0}(\mathbf{a})$ as follows

$$
\mathbf{I}_{\mathbf{L}}=\frac{\Omega_{\mathbf{0}}(\mathbf{a})}{\rho_{0}(\mathbf{a})} .
$$

From (7.12) it follows immediately for the vector $\mathbf{B} \equiv \Omega / \rho$ that

$$
\mathbf{B}(\mathbf{r}, t)=\hat{J} \mathbf{B}_{\mathbf{0}}(\mathbf{a}) \text {. }
$$

Thus, the Jacobi matrix becomes the evolution operator for the vector $\Omega / \rho$.

The invariants (7.12), indeed, are well-known in hydrodynamics but in a slightly different form. Let us write down the equation of motion for the fraction $\Omega / \rho$ which directly follows from Eqs. $(6.2,6.4)$,

$$
\frac{d}{d t} \mathbf{B}=(\mathbf{B} \nabla) \mathbf{v}
$$

Comparing this equation with the equation (6.8) for $\delta \mathbf{r}$ one can see that both quantities $\mathbf{B}$ and $\delta \mathbf{r}$ obey the same equation. This means that the vorticity is frozen into a fluid, the well-known statement in hydrodynamics. Sometimes this property is called as the frozenness of the vorticity into a fluid. If now one makes the next step, namely, multiplying Eq.(7.13) from the right by $\hat{J}^{-1}$ and Eq. (6.11) from the left by $\Omega / \rho$, after summation of the obtained results we arrive at the conservation of the vector invariant (7.12). These integrals just consist in the mathematical formulation of the frozenness of the vorticity into a fluid. The corresponding equation for the vector field $\mathbf{B}$ is called by the frozenness equation.

ii) In the general case (for arbitrary dependence of pressure on both density and entropy) from this vector invariant the only scalar that survives is a projection of $\mathbf{I}_{\mathbf{L}}$ to the vector $\nabla s$ :

$$
I_{L}=\left(\nabla_{a} s\left[\nabla_{a} \dot{x}_{i} \times \nabla_{a} x_{i}\right]\right) .
$$

Here all derivatives are taken with respect to a. Passing in this expression to the Eulerian variables and using the identity

$$
\epsilon_{\alpha \beta \gamma} \frac{\partial x_{i}}{\partial a_{\alpha}} \frac{\partial x_{j}}{\partial a_{\beta}} \frac{\partial x_{k}}{\partial a_{\gamma}}=\epsilon_{i j k} J
$$

one can get

$$
I_{L}=\frac{(\Omega \nabla s)}{\rho} .
$$


This integral is just the Ertel invariant (6.1). Thus, the conservation of the Ertel invariant as well as the Kelvin theorem about the conservation of the velocity circulation are a sequence of a specific gauge symmetry - the relabeling group.

It is interesting to follow how all the above formulas transform in two dimensions. In this case the Ertel invariant is identically equal to zero, due to the orthogonality of the vectors $\Omega$ and $\nabla s$. Therefore the nontrivial answers appear only for the barotropic fluid.

Applying the identity to (7.11) the identity

$$
\epsilon_{\alpha \beta} \frac{\partial x_{i}}{\partial a_{\alpha}} \frac{\partial x_{j}}{\partial a_{\beta}}=\epsilon_{i j} J
$$

it is easy to get that the Cauchy invariant transforms into the well-known Lagrangian invariant:

$$
\frac{\Omega}{\rho}=\operatorname{const}(a)
$$

Important to pay attention to that, unlike three-dimensional case, this relation does not contain the Jacobi matrix.

Let us turn to the incompressible fluid. In this case the obtained formulas are simplified. For example, the relation (7.12) in three dimensions is written in the form

$$
\mathbf{I}_{\mathbf{L}}=(\Omega, \nabla) \mathbf{a} \text {. }
$$

In the formula (7.14) $\mathbf{I}_{\mathbf{l}}$ coincides with

$$
\Omega_{\mathbf{0}}(\mathbf{a})=\operatorname{curl}_{a} \mathbf{u}
$$

where the vector $\mathbf{u}$ is defined by means of (4.19). This, in particular, means that the transverse part of the vector $\mathbf{u}$ conserves (being the Lagrangian invariant), and its temporal varying is due to its longitudinal part. Moreover, as pointed out in the fourth section, the choice of this vector is arbitrary due to the arbitrariness in the Lagrangian markers choice. The same takes place also to the vector $\Omega_{0}(\mathbf{a})$. If one performs the contact transformations $\mathbf{b}=\mathbf{b}(\mathbf{a})$ under the condition $\partial\left(b_{1} b_{2} b_{3}\right) / \partial\left(a_{1} a_{2} a_{3}\right)=1$, then the vector $\Omega_{0}(\mathbf{a})$ will be transformed by the law:

$$
\tilde{\Omega}_{0 i}(\mathbf{b})=\frac{\partial b_{i}}{\partial a_{j}} \Omega_{0 j}(\mathbf{a}) .
$$

This is the transformation of the gauge type, being the generalization [45] of the gauge transformations for the Clebsch variables (4.15) ${ }^{7}$.

Let, as a result of these transformations, the vector $\tilde{\Omega}_{\mathbf{0}}(\mathbf{b})$ have one nonzeroth component, say, z-component, equal to 1 :

$$
\begin{aligned}
& \tilde{\Omega}_{01}=\left(\tilde{\Omega}_{0} \nabla_{a}\right) b_{1}=0, \\
& \tilde{\Omega}_{02}=\left(\tilde{\Omega}_{0} \nabla_{a}\right) b_{2}=0, \\
& \tilde{\Omega}_{03}=\left(\tilde{\Omega}_{0} \nabla_{a}\right) b_{3}=1 .
\end{aligned}
$$

\footnotetext{
${ }^{7}$ Another approach to the gauge transformations in hydrodynamics was developed in [84].
} 
These relations within the given "vorticity" $\boldsymbol{\Omega}_{\mathbf{0}}(\mathbf{a})$ represent the equations to determine the dependence $\mathbf{b}(\mathbf{a})$. These are the linear differential equations of the first order, which allow to be applied the method of characteristics. Equations for characteristics here are the same for all three equations of the system (7.16),

$$
\frac{d \mathbf{a}}{d s}=\Omega_{\mathbf{0}}(\mathbf{a})
$$

that define the "vortex" line for the $\boldsymbol{\Omega}_{\mathbf{0}}(\mathbf{a})$. (Here $s$ may be understood as the arc length of the "vortex" line.) Equations on the characteristic (for the component of the $\mathbf{b}$ are then given by:

$$
\begin{aligned}
& \frac{d b_{1}}{d s}=0, \\
& \frac{d b_{2}}{d s}=0, \\
& \frac{d b_{3}}{d s}=1 .
\end{aligned}
$$

Two first components $b_{1}$ and $b_{2}$ are constant along the characteristic. Therefore $b_{1}$ and $b_{2}$ can be chosen as two independent integrals $c_{1}$ and $c_{2}$ of the system for characteristics (7.19), and the third component is a linear function of the arc length s. It is important to notice that a solution of the system (7.16) can be found always, at least, locally in the vicinity of some nonsingular surface provided by the coordinate system given by the constants $c_{1}$ and $c_{2}$. Rigorously speaking this is not a global solution as it is usual when one uses the method of characteristics.

Hence, by using the equation $\operatorname{curl}_{b} \tilde{\mathbf{u}}=\tilde{\Omega}_{\mathbf{0}}(\mathbf{b})$, one can reconstruct the velocity $\tilde{\mathbf{u}}:$

$$
\begin{aligned}
& \tilde{u}_{1}=\frac{\partial \phi}{\partial b_{1}}, \\
& \tilde{u}_{2}=\frac{\partial \phi}{\partial b_{2}}+b_{1}, \\
& \tilde{u}_{3}=\frac{\partial \phi}{\partial b_{3}} .
\end{aligned}
$$

After substitution of these expressions into the equation (4.19) we come back to the Clebsch representation with one pair of canonical variables (for more details, see [44]) which yields

$$
\mathbf{v}=b_{1} \nabla b_{2}+\nabla \phi .
$$

So, the vorticity $\Omega(\mathbf{r}, t)$ takes the form,

$$
\boldsymbol{\Omega}(\mathbf{r}, t)=\left[\nabla b_{1} \times \nabla b_{2}\right]=\frac{\partial \mathbf{r}}{\partial b_{3}}(\mathbf{b}, t) .
$$


The last equality is a direct sequence of the property following which the transformation $\mathbf{b}=\mathbf{b}(\mathbf{r}, t)$ is a diffeomorphism preserving the volume. It is easy to check also that the same expression as (7.25) where $\mathbf{r}$ is replaced by $\mathbf{b}$ satisfies the system (7.16). In this case the first equation of the system becomes the equation $\partial\left(b_{1} b_{2} b_{3}\right) / \partial\left(a_{1} a_{2} a_{3}\right)=1$.

Thus, locally any flow of incompressible fluid can be paratmerized by one pair of the Clebsch variables. In the general situation one needs two pairs of such variables.

\section{The Hopf Invariant and Degeneracy of the Pois- son Brackets}

So far we have not discussed the question of which classes of flows are described by the canonical variables introduced in the preceding sections.

To begin with, we consider this question for the example of an ideal incompressible fluid.

Let a flow be parameterized in terms of Clebsch variables in some one-connected domain,

$$
\mathbf{v}=\lambda \nabla \mu+\nabla \varphi
$$

Take some point inside this domain and draw through this point some closed curve. Starting from this point and constructing continiousely Clebsch variables on each peice of this curve we come back to the point. Generally speaking, Clebsch variables will take different values. Thus, Clebcsh variables will be multi-valued functions of space coordinates. One partial case of fluid flows with multi-valued Clebsch variables allows the following geometrical interpretation.

Consider a compact oriented two-dimensional manifold $M^{2}$ and suppose that $\lambda$ and $\mu$ are local coordinates on this manifold.

The gauge transformations associated with the nonuniqueness of choice of the Clebsch variables lead to the appearance of a whole family of gauge-equivalent mani-

folds, obtainable from one another by continuous deformations, preserving the surface element:

$$
d \lambda d \mu=d \lambda^{\prime} d \mu^{\prime}
$$

It is therefore sufficient to select from each of such a family one representative. For example, among the surfaces of genus zero having the same area, it is natural to select the sphere $S^{2}$.

It is easy to understand that the inverse image of any point of $M^{2}$ in $R^{3}$ is a closed curve coinciding with a vortex line. This follows directly from the expression for the curl of the velocity:

$$
\boldsymbol{\Omega}=\mathrm{curl} \mathbf{v}=[\nabla \lambda \times \nabla \mu] .
$$


The vortex line is the intersection of the two surfaces $\lambda(\mathbf{r})=$ const, $\mu(\mathbf{r})=$ const. If the variables $\lambda$ and $\mu$ are single-valued functions, then the manifold $M^{2}$ cannot be a closed surface of genus $g$. Then the flows given by such variables have no nodes. This fact can also be proved differently.

It is known [55],[56] that the degree of knottiness of a flow is characterized in ideal hydrodynamics by the conserved quantity

$$
I=\int(\mathbf{v}, \operatorname{curl} \mathbf{v}) d \mathbf{r}
$$

The conservation of this integral follows immediately from the Kelvin theorem. In order to illustrate this statement, following [56] we consider two closed vortex lines

$$
\boldsymbol{\Omega}=\int \kappa_{1} \mathbf{n}_{1} \delta\left(\mathbf{r}-\mathbf{l}_{1}\left(s_{1}\right)\right) d s_{1}+\int \kappa_{2} \mathbf{n}_{2} \delta\left(\mathbf{r}-\mathbf{l}_{2}\left(s_{2}\right)\right) d s_{2}
$$

where $\mathbf{n}_{1,2}$ are the tangents and $d s_{1,2}$ the arc elements of these curves.

Calculating the velocity circulation around the contours $\mathbf{r}=\mathbf{l}_{1}\left(\mathbf{s}_{1}\right)$ and $\mathbf{r}=\mathbf{l}_{2}\left(\mathbf{s}_{2}\right)$, we find

$$
\oint\left(\mathbf{v}, d \mathbf{l}_{1}\right)=m \kappa_{2}, \oint\left(\mathbf{v}, d \mathbf{l}_{2}\right)=m \kappa_{1}
$$

where $m$ is the linking number of these two curves. Multiplying the first equation by $\kappa_{2}$ and the second by $\kappa_{1}$, and adding the results, we get the integral $I$ :

$$
\int\left(\mathbf{v}, \kappa_{1} d \mathbf{l}_{1}+\kappa_{2} d \mathbf{l}_{2}\right)=\int(\mathbf{v}, \operatorname{curl} \mathbf{v}) d \mathbf{r}=2 m \kappa_{1} \kappa_{2} .
$$

This formula is generalized without difficulty to a vortex, and then to a continuous distribution. The conservation law (8.2) is valid not only for the infinite region but for the finite one when the vorticity lines are tangent to the boundary.

This integral is thus identically equal to zero for a flow with the trivial topology, in particular, for flows parameterized in terms of single-valued Clebsch variables.

We shall show that the Clebsch variables in the formulation (8.1) described knotted flows, and illustrate their topological meaning.

Suppose that the variables $\lambda$ and $\mu$ are local coordinates on $S^{2}$. In this case $\lambda$ and $\mu$ are expressed in terms of the polar and azimuthal angles, $\theta$ and $\varphi$, so that

$$
\boldsymbol{\Omega}=2 \mathrm{~A}[\nabla \cos \theta \times \nabla \varphi]
$$

where $A$ is a dimensional constant. Now the Clebsch variables are no longer singlevalued functions, and on a circuit around the $z$ axis the angle $\varphi$ acquires an addition $2 \pi$. It is also convenient to go over, in the expression for the vector field $\Omega$, from the angles $\theta$ and $\varphi$ to the $\mathbf{n}$-field $\left(\mathbf{n}^{2}=1\right)[60]$ :

$$
\Omega_{\alpha}=\varepsilon_{\alpha \beta \gamma}\left(\mathbf{n},\left[\partial_{\beta} \mathbf{n} \times \partial_{\gamma} \mathbf{n}\right]\right) .
$$


We shall limit our considerations to the flows for which $\mathbf{n}$ tends sufficiently rapidly at the infinity to a constant vector $\mathbf{n}_{0}$. For this class on flows $R^{3}$ is isomorphic to the four-dimensional sphere $S^{3}$. Thus the classification of the flows is a problem of classification of smooth mappings $S^{3} \rightarrow S^{2}$. Such mappings are characterized by the homotopy group $\pi_{3}\left(S^{2}\right)=$ Z, i.e., any class of flows is characterized by the linked number that coincides with the winding number of two any lines $\mathbf{n}(\mathbf{r})=\mathbf{n}_{1}$ and $\mathbf{n}(\mathbf{r})=\mathbf{n}_{2}\left(\mathbf{n}_{1,2}=\right.$ const $)$. The index $N$ for smooth mappings is called the Hopf invariant [61]. One can show that the Hopf invariant coincides with the integral $I$ up to a constant factor [62]:

$$
I=\int(\mathbf{v}, \mathbf{\Omega}) d \mathbf{r}=64 \pi^{2} N A^{2} .
$$

The derivation of this relation is based on the well-known formula of Gauss for the linking number of two curves.

It should be mentioned that in the quantum case, according to [62], $A=\hbar / 2 m$. The remaining manifolds are od secondary interest from the point of view of topology. So a manifold $M^{2}$, which is a surface with boundary, is homotopically to a bouquet of circles. Therefore its homotopic group $\pi_{3}$ is trivial. The groups $\pi_{3}$ are also trivial for closed surfaces of genus $g \geq 1$. Topological nontrivial situations occur only for surfaces with zero genus.

We now give an example of a nontrivial mapping with $N=1$ (Hopf mapping):

$$
\begin{gathered}
(\mathbf{n}, \sigma)=q^{+} \sigma_{3} q, \\
q=(1-i \mathbf{r} \sigma)(1+i \mathbf{r} \sigma)^{-1}
\end{gathered}
$$

where $\sigma$ are the Pauli matrices.

In toroidal coordinates, one has

$$
\begin{gathered}
x+i y=\frac{\sinh U}{\cosh U+\cos \beta} e^{i \alpha}, z=\frac{\sin \alpha}{\cosh U+\cos \beta}, \\
(0 \leq U<\infty, 0<\alpha, \beta<2 \pi),
\end{gathered}
$$

and Eq. (8.4) reads

$$
\arctan \left(n_{y} / n_{x}\right)=\alpha-\beta, n_{z}=1-2 \tanh ^{2} U .
$$

These formulas show that the flow looks as follows: the whole space is sliced up by the tori $U=$ const, while any vortex line coils up on a torus, making one loop. Thus any vortex line links once. The expressions for $\Omega$ and $\mathbf{v}$, calculated from (8.4) are not solution of the stationary Euler equations, and can therefore be used as initial conditions for (5.9). It is obvious that the evolution of such a distribution does not take the solution out of the given class with Hopf invariant $N=1$. The evolution of the vector field $\mathbf{n}$ is determined from the equation

$$
\mathbf{n}_{t}+(\mathbf{v} \nabla) \mathbf{n}=0
$$


which is equivalent to the evolution equation for the variables $\lambda$ and $\mu$. Equations (8.5) are also Hamiltonian,

$$
\mathbf{n}_{\mathbf{t}}=2 A\left[\mathbf{n} \times \frac{\delta H}{\delta \mathbf{n}}\right]
$$

and differ from the familiar Landau-Lifshitz equations only by the choice the Hamiltonian $H$.

The Poisson bracket in this case coincides with the BKK bracket $(2.8),(2.11)$ :

$$
\{F, G\}=2 A \int\left(\mathbf{n}\left[\frac{\delta F}{\delta \mathbf{n}} \times \frac{\delta H}{\delta \mathbf{n}}\right]\right) d \mathbf{r} .
$$

When we go over in this bracket from the $\mathbf{n}$-field to $\Omega$ according to formula (8.3) we get the Poisson bracket (5.8). It is important to note that (5.8) is a degenerate bracket with respect to the invariant $I:\{I, \ldots\}=0$, which again shows its origin. From one side, it is connected with its topology, from another side, with the Kelvin theorem. One should remind that the latter is a sequence of the gauge symmetry of the Lagrangian markers.

As we see below, the question about degeneracy of the Poisson brackets for arbitrary equation of state directly is connected the gauge symmetry.

Let us discuss in more details this question for the Poisson hydrodynamic brackets. For this aim, we consider the most general form of the bracket for ideal hydrodynamics, namely, for the nonbarotropic fluids. The bracket in this case has the form of (5.6)

$$
\begin{gathered}
\{F, G\}=\int\left\{\left(\nabla \frac{\delta F}{\delta \rho}, \frac{\delta G}{\delta \mathbf{v}}\right)-\left(\nabla \frac{\delta G}{\delta \rho}, \frac{\delta F}{\delta \mathbf{v}}\right)\right\} d \mathbf{r} \\
+\int\left(\frac{\operatorname{curl} \mathbf{v}}{\rho},\left[\frac{\delta F}{\delta \mathbf{v}} \times \frac{\delta G}{\delta \mathbf{v}}\right]\right) d \mathbf{r}+\int\left(\frac{\nabla S}{\rho},\left[\frac{\delta F}{\delta \mathbf{v}} \frac{\delta G}{\delta S}-\frac{\delta G}{\delta \mathbf{v}} \frac{\delta F}{\delta S}\right]\right) d \mathbf{r} .
\end{gathered}
$$

By substituting the integral (7.1), $I_{i}=\int \rho f\left(I_{l}, S\right) d \mathbf{r}$, into this expression one can verify that the integral commutes with any functional,

$$
\left\{I_{i}, \cdot\right\}=0
$$

In accordance with the definition of the section 1, this integral represents the Casimir against the bracket (8.6).

One should remind that the fact of the conservation of the integral (7.1) is a sequence of the special gauge symmetry of the ideal hydrodynamics equations, that is, as we see, responsible also or the degeneracy of the Poisson brackets.

In order to transform from this bracket to the canonical one it is necessary to resolve the integral (7.1) by introducing new coordinates. We have already known one answer to the question how to do it. If we take the expression (4.17) for the velocity 
and put there instead of $\mu$ the Ertel invariant $I_{l}$ then the integral (7.1) transforms into the dynamical conservation law with respect to the canonical bracket

$$
\{F, G\}=\int\left\{\left[\frac{\delta F}{\delta \rho} \frac{\delta G}{\delta \varphi}-\frac{\delta F}{\delta \varphi} \frac{\delta G}{\delta \rho}\right]+\left[\frac{\delta F}{\delta \lambda} \frac{\delta G}{\delta I_{l}}-\frac{\delta F}{\delta I_{l}} \frac{\delta G}{\delta \lambda}\right]+\left[\frac{\delta F}{\delta \beta} \frac{\delta G}{\delta s}-\frac{\delta F}{\delta s} \frac{\delta G}{\delta \beta}\right]\right\} d \mathbf{r}
$$

so that

$$
\left\{I_{i}, H\right\}=0 .
$$

We can also remark that, as it was shown by van Saarlos [52], the transition from the Lagrangian description in terms of the action (7.6) to the canonical variables is determined through the change (9.11) or (4.20).

\section{Inhomogeneous Fluid and Surface Waves}

In this section we introduce canonical coordinates for the description of nonlinear waves in an ideal fluid of variable density. Here one distinguishes two types of waves. The first type refers to so-called internal waves, propagating in a continuous medium with a smooth inhomogeneity. The second type refers to the situation where the density gradient changes sharply over the size of the wave length, and in the limit represents simply a jump. In this limit we talk about surface waves. Canonical variables can be introduced in both cases within the framework of the scheme developed in the preceding sections.

Consider an ideal fluid of varying density in the presence of a constant gravitational field $\mathbf{g}$ anti-parallel to the $z$ axis. The fluid is assumed to be locally incompressible. This means that the density is convected along the fluid and is therefore a Lagrange variable:

$$
\frac{\partial \rho}{\partial t}+(\mathbf{v} \nabla) \rho=0 \text { for } \operatorname{div} \mathbf{v}=0
$$

These two equations therefore appear in the Lagrangian as constraints:

$$
L=\int\left[\rho \frac{\mathbf{v}^{2}}{2}-U(\rho, \mathbf{r})-\alpha\left(\frac{\partial \rho}{\partial t}+(\mathbf{v} \nabla) \rho\right)+\varphi \operatorname{div} \mathbf{v}\right] d \mathbf{r}
$$

where $U(\rho, \mathbf{r})$ is the density of potential energy in the presence of the field $\mathbf{g}$, given by the expression

$$
U(\rho, \mathbf{r})=g\left[\rho\left(\mathbf{r}_{\perp}, z\right)\left(z-z^{\prime}\right)-\int_{z^{\prime}}^{z} \rho_{0}\left(z^{\prime \prime}\right) d z^{\prime \prime}\right]
$$

The first term in this expression corresponds to the work in lifting the fluid element to the point $z$ from the equilibrium point $z^{\prime}$, determined from the condition 
for equality of the equilibrium density $\rho_{0}\left(z^{\prime}\right)$ and the density of fluid at the given point,

$$
\rho_{0}\left(z^{\prime}\right)=\rho\left(\mathbf{r}_{\perp}, z\right) .
$$

This relation gives $z^{\prime}$ as a function of the density, $z^{\prime}=z^{\prime}(\rho)$. The second term in (9.2) corresponds to the potential of the Archimedean force.

Variations of the Lagrangian with respect to $\mathbf{v}$ and $\varphi$ lead us to the equations $[26]$ :

$$
\rho \mathbf{v}=\nabla \varphi+\alpha \nabla \rho \text { for } \operatorname{div}\left[\rho^{-1}(\nabla \varphi+\alpha \nabla \rho)\right]=0,
$$

giving the connection between the new and old variables. Varying with respect to variable $\rho$, we get an equation for the potential $\alpha$ :

$$
\frac{\partial \alpha}{\partial t}+(\mathbf{v} \nabla) \alpha+\frac{\mathbf{v}^{2}}{2}-\frac{\partial U}{\partial \rho}=0
$$

where $\partial U / \partial \rho=g\left(z-z^{\prime}\right)$.

Next, substituting (9.3) in the Euler equation (4.8) and using the equations of motion for $\alpha$ and $\rho$, we obtain up to constant an expression for the pressure $p$, analogous to the Bernoulli integral:

$$
p=-\rho \frac{\mathbf{v}^{2}}{2}-\rho g\left(z-z^{\prime}\right)+\left(\frac{\partial}{\partial t}+(\mathbf{v} \nabla)\right) \varphi+\text { const }
$$

The Hamiltonian is formed in the standard way and coincides with the total energy

$$
H=\int\left[\rho \frac{\mathbf{v}^{2}}{2}+U(\rho, \mathbf{r})\right] d \mathbf{r},
$$

while the variables $\alpha$ and $\rho$ occur to be canonically conjugate:

$$
\frac{\partial \alpha}{\partial t}=\frac{\delta H}{\delta \rho}, \frac{\partial \rho}{\partial t}=-\frac{\delta H}{\delta \alpha} .
$$

The parameterization, presented here, for the velocity in terms of the density $\rho$ and $\alpha$ imposes strong restrictions on the form of the initial distribution. As we see from (9.3), the curl of the mass current at all times, including the initial time, is orthogonal to the density gradient. Such motions are the analog of potential motions in a homogeneous fluid. This scheme can considerably be improved if one includes "nonpotential" motions. As far as the noncanonical Poisson brackets concern, they were introduced in the paper [64].

If one needs to consider weakly nonlinear oscillations in a stratified fluid one should expand the Hamiltonian in powers of $\alpha$ and $\delta \rho$. In particular, the well-known Boussinesq approximation is obtained if the density in the kinetic energy is replaced by some averaged constant quantity. 
Now let us consider one important limiting case of a stratified fluid, when the stratification is only due to a free boundary.

First we look at potential motions. For them the Lagrangian has the same form as before, in which the density should be regarded as constant throughout the volume of the fluid, i.e.,

$$
\rho=\rho_{0} \theta\left(z-\eta\left(\mathbf{r}_{\perp}, t\right)\right) .
$$

Here $\theta(z)$ is the Heaviside function, and $\eta\left(\mathbf{r}_{1}, t\right)$ is the deviation of the free surface from the horizontal axis $z=0$. The element of free surface $d s_{n}=d \mathbf{r}_{\perp} \sqrt{1+(\nabla \eta)^{2}}$ and the vector normal to it, $\mathbf{n}=(-\nabla \eta)\left[1+(\nabla \eta)^{2}\right]^{-1 / 2}$, are expressed in terms of the function $\eta\left(\mathbf{r}_{\perp}\right)$ explicitly, as is the potential energy

$$
U=\int\left\{\frac{\rho_{0} g \eta^{2}}{2}+\sigma\left[\sqrt{1+(\nabla \eta)^{2}}-1\right]\right\} d \mathbf{r}_{\perp}
$$

in which we have taken into account the surface tension with coefficient $\sigma$.

It is easy to see that the continuity equation in the present case becomes the kinematic condition

$$
\frac{d \eta}{d t}=\left(\frac{\partial}{\partial t}+\mathbf{v} \nabla\right) \eta=0
$$

In accordance with this, the Lagrangian expresses

$$
L=\int d \mathbf{r}_{\perp} \int_{-h}^{\eta} d z\left(\frac{\rho_{0} \mathbf{v}^{2}}{2}+\varphi \operatorname{div} \mathbf{v}\right)+\int \psi\left(\frac{\partial \eta}{\partial t}-v_{n} \sqrt{1+(\nabla \eta)^{2}}\right) d \mathbf{r}_{\perp}-U
$$

Here

$$
v_{n}=\frac{\left[v_{z}-\mathbf{v} \nabla \eta\right]_{z=\eta}}{\sqrt{1+(\nabla \eta)^{2}}}
$$

is the normal component of the velocity and $\psi=-\alpha \rho_{0}$ the Lagrange multiplier given on the free surface. Variation of $L$ with respect to $\mathbf{v}$ within the bulk leads to the potential equation $\rho_{0} \mathbf{v}=\nabla \varphi$ where $\varphi$ is determined from the solution of the Laplace equation $\Delta \varphi=0$. Variation of $L$ with respect to $\mathbf{v}$ on the boundary (for fixed $\eta$ ) gives the boundary conditions for the Laplace equation

$$
\left.\varphi\right|_{z=\eta}=\psi
$$

The variation of the Lagrangian with respect to $\eta$ is nontrivial. For this it is convenient to rewrite all the terms in (9.5) containing $\mathbf{v}$ in the form of a volume integral which we designate as $L_{v}$. Then, taking (9.6) into account, we have

$$
L_{v}=\int d \mathbf{r}_{\perp} \int_{-h}^{\eta} d z\left(\frac{\rho_{o} \mathbf{v}^{2}}{2}-\mathbf{v} \nabla \varphi\right) .
$$


The variation $\delta L_{v}$ for a change in $\eta$ is composed of two terms. The first is caused by the volume change:

$$
\int d \mathbf{r}_{\perp}\left(\frac{\rho_{0} \mathbf{v}^{2}}{2}-\mathbf{v} \nabla \varphi\right) \delta \eta
$$

The second arises when we consider variations of $\mathbf{v}$ and $\varphi$, not caused by the change in shape of these functions, for example,

$$
\delta \varphi=\varphi(z-\delta \eta)-\varphi(z)=-\frac{\partial \varphi}{\partial z} \delta \eta
$$

Therefore the contribution to $\delta L_{v}$ of this variation has the form

$$
\int d \mathbf{r}_{\perp} v_{n} \sqrt{1+(\nabla \eta)^{2}} \frac{\partial \varphi}{\partial z} \delta \eta
$$

Collecting all terms together we finally get

$$
\begin{gathered}
\frac{\partial \psi}{\partial t}=-\rho_{0} g \eta+\sigma \operatorname{div} \frac{\nabla \eta}{\sqrt{1+(\nabla \eta)^{2}}} \\
+\left[\frac{\rho_{0} \mathbf{v}^{2}}{2}-\mathbf{v} \nabla \varphi+\frac{\partial \varphi}{\partial z} v_{n} \sqrt{1+(\nabla \eta)^{2}}\right]_{z=\eta} .
\end{gathered}
$$

The Hamiltonian $H$, as before, coincides with the total energy of the system,

$$
H=\int d \mathbf{r}_{\perp} \int_{-n}^{\eta} d z \frac{\rho_{0}(\nabla \varphi)^{2}}{2}+U
$$

while the Hamilton equation has the form [25]:

$$
\frac{\partial \eta}{\partial t}=\frac{\delta H}{\delta \psi}, \quad \frac{\partial \psi}{\partial t}=-\frac{\delta H}{\delta \eta}
$$

Let us now consider the expansion of the Hamiltonian $H$ in powers of the canonical variables. In the coordinate representation each term in this series is a nonlocal functional of $\eta$ and $\psi$; the reason for this is that at each step of the iteration we must solve the Laplace equation. After applying Fourier transformation with respect to the coordinates in the horizontal plane and successive approximations, one can get

$$
\begin{aligned}
H & =\frac{1}{2} \int\left(g+\sigma k^{2}\right)\left|\eta_{k}\right|^{2} d \mathbf{k}+\frac{1}{2} \int k \tanh (k h) \psi\left|\psi_{k}\right|^{2} d \mathbf{k} \\
& +\frac{1}{2 \cdot 2 \pi} \int L_{k k_{1} k_{2}} \psi_{k} \psi_{k_{1}} \eta_{k_{2}} \delta_{k+k_{1}+k_{2}} d \mathbf{k} d \mathbf{k}_{1} d \mathbf{k}_{2}+\ldots,
\end{aligned}
$$

where

$$
L_{k k_{1} k_{2}}=\frac{1}{2}\left(k^{2}+k_{1}^{2}-k_{2}^{2}\right)-k k_{1} \tanh (k h) \tanh \left(k_{1} h\right), \quad\left(\rho_{0}=1\right) .
$$


The expansion in (9.8) is performed with respect to the parameter $k \eta$, having the significance of a characteristic angle of inclination of the fluid surface.

In the limit of shallow water $k h \rightarrow 0$, the above expression reduces to

$$
L_{k k_{1} k_{2}} \rightarrow-\left(\mathbf{k k}_{1}\right)
$$

i.e., the cubic term of the expansion $H_{1}$ becomes local in the variables $\psi$ and $\eta$ :

$$
H_{1}=\frac{1}{2} \int \eta(\nabla \psi)^{2} d \mathbf{r}_{1}
$$

In particular, the transition to the known Boussinesq model (cf., for example,[67]) is accomplished if we take (9.9) for the interaction Hamiltonian, while we include in $H_{0}$ the terms proportional to $h^{3}$ :

$$
\begin{gathered}
\frac{\partial \eta}{\partial t}=-h \Delta \psi-\operatorname{div} \eta \nabla \psi+\frac{h^{3}}{3} \Delta^{2} \psi=\frac{\delta H}{\delta \psi} \\
\frac{\partial \psi}{\partial t}=-g \eta+\sigma \Delta \eta-\frac{(\nabla \psi)^{2}}{2}=-\frac{\delta H}{\delta \eta}
\end{gathered}
$$

where

$$
H=\frac{1}{2} \int\left\{g \eta^{2}+\sigma(\nabla \eta)^{2}+h(\nabla \psi)^{2}-h^{3}(\Delta \psi)^{2}+\eta(\nabla \psi)^{2}\right\} d \mathbf{r}_{1}
$$

In the limit of deep water, $L_{k k_{1} k_{2}}$ behaves like

$$
L_{k k_{1} k_{2}} \rightarrow-\left(\mathbf{k k}_{1}\right)-k k_{1} .
$$

The transition to normal variables is given by the formulas

$$
\begin{aligned}
\eta_{k} & =\left(\frac{\omega_{k}}{2\left(g+\sigma k^{2}\right.}\right)^{1 / 2}\left(a_{k}+a_{-k}^{*}\right), \\
\psi_{k} & =-i\left(\frac{g+\sigma k^{2}}{2 \omega_{k}}\right)^{1 / 2}\left(a_{k}-a_{-k}^{*}\right),
\end{aligned}
$$

where $\omega_{k}=\left[k\left(g+\sigma k^{2}\right) \tanh (k h)\right]^{1 / 2}$ is the dispersion law for surface waves.

In the same spirit as this was done in Section 4, one can include the contribution from nonpotential flows [1]. For this it is necessary to involve an additional constraint in the Lagrangian,

$$
\frac{\partial \mu}{\partial t}+\mathbf{v} \nabla \mu=0
$$

so that the Lagrangian has the form,

$$
L=\int d \mathbf{r}_{\perp} \int_{-h}^{\eta} d z\left(\frac{\rho_{0} \mathbf{v}^{2}}{2}+\varphi \operatorname{div} \mathbf{v}-\lambda\left(\mu_{t}+\mathbf{v} \nabla \mu\right)\right)
$$




$$
+\int \psi\left(\frac{\partial \eta}{\partial t}-v_{n} \sqrt{1+(\nabla \eta)^{2}}\right) d \mathbf{r}_{\perp}-U
$$

With such a choice for the Lagrangian, $\mathbf{v}$ is given, as in Section 4 , in terms of the Clebsch variables $\lambda$ and $\mu$ :

$$
\rho_{0} \mathbf{v}=\lambda \nabla \mu+\nabla \varphi .
$$

whose equations have been derived in (4.9) and (4.13). The function $\psi$, as for a potential flow, has the same meaning

$$
\left.\varphi\right|_{z=\eta}=\psi \text {. }
$$

The equation for this value takes the form of (9.7) where the velocity $\mathbf{v}$ is replaced by the expression (9.11).

Another way to introduce "surface" canonical variables is given in [23]. In a similar way we can introduce canonical variables into a stratified fluid, taking into account the "nonpotential" variables $\lambda$ and $\mu$.

Noncanonical Poisson brackets for the case of arbitrary flows bounded by a free surface were introduced in the paper [65]. The bracket represents the combination of the Zakharov's bracket [24],[25] for potential flow and the bracket (5.7):

$$
\{F, G\}=\int\left(\operatorname{curl} \mathbf{v},\left[\frac{\delta F}{\delta \mathbf{v}} \times \frac{\delta G}{\delta \mathbf{v}}\right]\right) d \mathbf{r}+\int_{\Sigma}\left(\frac{\delta F}{\delta \Sigma} \frac{\delta G}{\delta \psi}-\frac{\delta G}{\delta \Sigma} \frac{\delta F}{\delta \psi}\right) d s .
$$

Here $F$ and $G$ are functionals of the velocity $\mathbf{v}($ divv $=0)$ and the free surface $\Sigma$, ds the surface element. The variational derivatives of $\delta F / \delta \mathbf{v}$ and $\delta G / \delta \mathbf{v}$ are divergence free. The potential part of the velocity is introduced by the velocity unique decomposition (for more details, see [66])

$$
\mathbf{v}=\mathbf{w}+\nabla \Phi
$$

where $\mathbf{w}$ is divergence free and tangent to $\Sigma$. The potential $\Phi$ is determined by the equations,

$$
\Delta \Phi=0, \frac{\partial \Phi}{\partial n}=v_{n}
$$

In Eq. (9.13) $\psi$ is the limit of $\Phi$ on the free surface $\Sigma$.

The equations of motion then read

$$
\frac{\partial \mathbf{v}}{\partial t}+(\mathbf{v}, \nabla) \mathbf{v}=-\nabla p, \frac{\partial \Sigma}{\partial t}=v_{n}
$$

with two conditions

$$
\operatorname{div} \mathbf{v}=0,\left.p\right|_{\Sigma}=\sigma \kappa
$$

where $\kappa$ is the mean curvature of the free surface. By means of the bracket (9.13), they can be written in the form

$$
\frac{\partial \mathbf{v}}{\partial t}=\{\mathbf{v}, H\}, \quad \frac{\partial \Sigma}{\partial t}=\{\Sigma, H\}
$$


There is also no difficulty in introducing canonical variables for the description of interacting internal and surface waves. For this case the Lagrangian is a combination of the Lagrangians (9.1) and (9.5).

We would like to mention the interesting paper [70] where the canonical Hamiltonian approach was developed for the description of the interaction of surface waves and vortex filaments. The canonical variables introduced in this paper can be extracted from the general noncanonical Poisson brackets (9.13) by the corresponding limit to the vortex filament.

The introduction of canonical variables for internal and surface waves is also possible for more complicated systems, for example, for a dielectric fluid in an external electric field or a ferro-fluid in a magneto-static field [69]. For these systems the Hamiltonian coincides with the free energy in the external electric (magnetic) field, and the canonical variables remain the same as in the absence of the field.

\section{Hamiltonian Formalism for Plasma and Mag- netohydrodynamics}

The simplest hydrodynamic models of a plasma belong to the type of (4.1), (4.2). Let us consider the hydrodynamics of electrons interacting with a potential electric field in a plasma without magnetic field:

$$
\begin{gathered}
\frac{\partial \rho}{\partial t}+\operatorname{div} \rho \mathbf{v}=0 \\
\frac{\partial \mathbf{v}}{\partial t}+(\mathbf{v} \nabla) \mathbf{v}=-\nabla\left[\frac{e}{m} \varphi+\frac{3 T}{m \rho_{0}} \delta \rho\right] \\
\Delta \varphi=-4 \pi e \frac{\delta \rho}{m}, \delta \rho=\rho-\rho_{0} .
\end{gathered}
$$

Here $e$ and $m$ are the electron charge and mass, respectively, and $T$ is the temperature.

The internal energy of such a system is composed of the electrostatic energy

$$
\mathcal{E}_{e s}=\frac{1}{8 \pi} \int(\nabla \varphi)^{2} d \mathbf{r}=\frac{e^{2}}{2 m^{2}} \int \frac{\delta \rho(\mathbf{r}) \delta \rho\left(\mathbf{r}^{\prime}\right)}{\left|\mathbf{r}-\mathbf{r}^{\prime}\right|} d \mathbf{r} d \mathbf{r}^{\prime}
$$

and the gas-kinetic energy

$$
\mathcal{E}_{T}=\frac{3}{2} \frac{T}{m \rho_{0}} \int \delta \rho^{2} d \mathbf{r}
$$

It is obvious that

$$
\frac{e}{m} \varphi=\frac{e^{2}}{m^{2}} \int \frac{\delta \rho\left(\mathbf{r}^{\prime}\right)}{\left|\mathbf{r}-\mathbf{r}^{\prime}\right|} d \mathbf{r}=\frac{\delta \mathcal{E}_{e s}}{\delta \rho}
$$




$$
\frac{3 T}{m \rho_{0}} \delta \rho=\frac{\delta \mathcal{E}_{T}}{\delta \rho} .
$$

Formula (10.16) shows that the system (10.14) belongs to the type of (4.1), (4.2) with $\mathcal{E}_{\text {in }}$ in the general form (4.6). The diagonalizing transformation for $H_{0}$ in this case has the form (4.4), in which one should set $\omega_{k}^{2}=\omega_{p}^{2}+3 k^{2} T / m\left(\omega_{p}^{2}=4 \pi_{0} e^{2} / m^{2}\right)$ while the coefficients $U$ and $V$ are determined from formulas (4.5), in which we should take $g=0$.

Now let us consider the hydrodynamics of slow motion of a nonisothermal plasma, whose electron temperature $T_{e}$ significantly exceeds the ion temperature. By slow motion we shall understand wave motion with phase velocities $\omega / k$ much smaller than the electron thermal velocity, but large compared to the ion thermal velocity. In this case we can assume that the electrons are distributed according to the Boltzmann law, $\rho_{e}=\rho_{0} e^{e \varphi / T_{e}}$, while the ion thermal motion can be neglected. Then:

$$
\begin{aligned}
\frac{\partial \rho}{\partial t}+\operatorname{div} \rho \mathbf{v} & =0, \frac{\partial \mathbf{v}}{\partial t}+(\mathbf{v} \nabla) \mathbf{v}=-\frac{e}{M} \nabla \varphi, \\
\Delta \varphi & =\frac{4 \pi e}{M}\left(\rho-\rho_{0} e^{e \varphi / T_{e}}\right),
\end{aligned}
$$

where $M$ is the ion mass.

This system also conserves the energy

$$
H=\int \frac{\rho \mathbf{v}^{2}}{2} d \mathbf{r}+\mathcal{E}_{i n} .
$$

Here $\mathcal{E}_{\text {in }}$ is the internal energy, equal to the sum of the electrostatic energy $\mathcal{E}_{e s}=$ $(1 / 8 \pi) \int(\nabla \varphi)^{2} d \mathbf{r}$ and the thermal energy of the electron gas

$$
\mathcal{E}_{T}=\frac{T_{e}}{M} \int \rho_{0}\left\{e^{e \varphi / T_{e}}\left(\frac{e \varphi}{T_{e}}-1\right)+1\right\} d \mathbf{r} .
$$

Calculating the variational derivative of $\mathcal{E}_{\text {in }}$ with respect to the ion density $\rho$, we get

$$
\frac{\delta \mathcal{E}_{i n}}{\delta \rho}=\int \varphi\left(\mathbf{r}^{\prime}\right)\left\{-\frac{1}{4 \pi} \Delta \frac{\delta \varphi\left(\mathbf{r}^{\prime}\right)}{\delta \rho(\mathbf{r})}+\frac{e^{2} \rho_{0}}{M} e^{e \varphi / T e} \frac{\delta \varphi\left(\mathbf{r}^{\prime}\right)}{\delta \rho(\mathbf{r})}\right\} d \mathbf{r}^{\prime} .
$$

On the other hand, by varying the Poisson equation we have:

$$
-\frac{1}{4 \pi} \Delta \frac{\delta \varphi\left(\mathbf{r}^{\prime}\right)}{\delta \rho(\mathbf{r})}+\frac{e^{2} \rho_{0}}{M} e^{e \varphi / T e} \frac{\delta \varphi\left(\mathbf{r}^{\prime}\right)}{\delta \rho(\mathbf{r})}=\frac{e}{M} \delta\left(\mathbf{r}-\mathbf{r}^{\prime}\right) .
$$

Comparing the two expressions, we arrive at

$$
\frac{\delta \mathcal{E}_{\text {in }}}{\delta \rho}=\frac{e}{M} \varphi .
$$


From this it follows that the system (10.18) also belongs to type (4.1), (4.2).

We note that for long-wave $\left(k r_{d} \ll 1\right.$, where $r_{d}=\left(T e / 4 \pi n_{0} e^{2}\right)^{1 / 2}$ is the Debye radius) oscillations of small amplitude, the system of Boussinesq equations follows from (10.18). It is easy to see that in this limit the potential is determined from the Poisson equation,

$$
\frac{e \varphi}{T e} \approx \frac{\delta \rho}{\rho_{0}}-\frac{1}{2}\left(\frac{\delta \rho}{\rho_{0}}\right)^{2}+r d^{2} \Delta \frac{\delta \rho}{\rho_{0}} .
$$

Substitution of this expression in (10.19) leads to the following form for the internal energy (cf. (4.6)),

$$
\mathcal{E}_{i n}=\int \frac{\rho_{0} c_{s}^{2}}{2}\left[\left(\frac{\delta \rho}{\rho_{0}}\right)^{2}-\frac{1}{3}\left(\frac{\delta \rho}{\rho_{0}}\right)^{3}-r d^{2}\left(\nabla \frac{\delta \rho}{\rho_{0}}\right)^{2}\right] d \mathbf{r}, c_{s}^{2}=T_{e} / M
$$

We now go over to a consideration of the relativistic gas dynamics of electrons, interacting with an arbitrary, nonpotential electromagnetic field:

$$
\begin{gathered}
\frac{\partial \rho}{\partial t}+\operatorname{div} \rho \mathbf{v}=0 \\
\left(\frac{\partial}{\partial t}+\mathbf{v} \nabla\right) \mathbf{p}=e \mathbf{E}+\frac{e}{c}[\mathbf{v} \times \mathbf{H}]-3 T \nabla \frac{\delta \rho}{\rho_{0}}, \\
\operatorname{curl} \mathbf{E}=-\frac{1}{c} \frac{\partial \mathbf{H}}{\partial t}, \operatorname{curl} \mathbf{H}=\frac{4 \pi}{c} \frac{e \rho}{m} \mathbf{v}+\frac{1}{c} \frac{\partial \mathbf{E}}{\partial t}, \\
\operatorname{div} \mathbf{E}=4 \pi e \delta \rho / m .
\end{gathered}
$$

For the electromagnetic field we introduce scalar and vector potentials $\varphi$ and $\mathbf{A}$, where we choose for $\mathbf{A}$ the Coulomb gauge, $\operatorname{div} \mathbf{A}=0$.

We know that in the Coulomb gauge the vector potential is a canonical variable, if we change from ordinary momentum to generalized momentum,

$$
\mathbf{p}=\mathbf{p}_{1}-\frac{e}{c} \mathbf{A}
$$

determined from the equation:

$$
\frac{\partial \mathbf{p}_{1}}{\partial t}+\nabla\left(m^{2} c^{4}+p^{2} c^{2}\right)^{1 / 2}-\left[\mathbf{v} \times \operatorname{curl} \mathbf{p}_{1}\right]+e \nabla \varphi=-3 T \nabla \frac{\delta \rho}{\rho_{0}} .
$$

The canonical conjugate of $\mathbf{A}$ is the vector

$$
\mathbf{B}=\frac{1}{4 \pi c}\left(\nabla \varphi+\frac{1}{c} \frac{\partial \mathbf{A}}{\partial t}\right)=-\frac{\mathbf{E}}{4 \pi c} .
$$


The other variables are introduced by analogy with the Clebsch variables:

$$
\frac{\mathbf{p}_{1}}{m}=\frac{\lambda}{\rho} \nabla \mu+\nabla \varphi .
$$

Here $(\lambda, \mu),(\rho, \varphi),(\mathbf{B}, \mathbf{A})$ are canonically conjugate quantities,

$$
\begin{gathered}
\frac{\partial \lambda}{\partial t}=\frac{\delta H}{\delta \mu}, \frac{\partial \mu}{\partial t}=-\frac{\delta H}{\delta \lambda}, \frac{\partial \rho}{\partial t}=\frac{\delta H}{\delta \varphi}, \frac{\partial \varphi}{\partial t}=-\frac{\delta H}{\delta \rho}, \\
\frac{\partial \mathbf{A}}{\partial t}=\frac{\delta H}{\delta \mathbf{B}}, \frac{\partial \mathbf{B}}{\partial t}=-\frac{\delta H}{\delta \mathbf{A}}
\end{gathered}
$$

with the Hamiltonian

$$
\begin{gathered}
H=\int\left[\frac{\rho}{m}\left(p^{2} c^{2}+m^{2} c^{4}\right)^{1 / 2}+\frac{3}{2} T \frac{\delta \rho^{2}}{m \rho_{0}}+\frac{1}{8 \pi}(\operatorname{curl} \mathbf{A})^{2}\right] d \mathbf{r} \\
+\int\left[2 \pi c^{2} B^{2}-c(\mathbf{B} \nabla \varphi)+\frac{1}{4 \pi} \varphi \Delta \varphi\right] d \mathbf{r}
\end{gathered}
$$

coinciding with the total energy of the system if the Poisson equation is satisfied identically.

We note that canonical variables are introduced analogously for the two-fluid model of the plasma. A more detailed presentation of these results can be found in the papers $[34,35]$.

Another widely used model in plasma physics is the set of magnetohydrodynamic (MHD) equations, describing low-frequency (hydrodynamic) motions of the plasma as a whole. These equations, in particular, can be obtained from the equations of the two-fluid model.

For barotropic flows, the equations of MHD have the form

$$
\begin{gathered}
\frac{\partial \rho}{\partial t}+\operatorname{div} \rho \mathbf{v}=0 \\
\frac{\partial \mathbf{v}}{\partial t}+(\mathbf{v} \nabla) \mathbf{v}=-\nabla \frac{\delta \varepsilon}{\delta \rho}+\frac{1}{4 \pi \rho}[\operatorname{curl} \mathbf{H} \times \mathbf{H}], \\
\frac{\partial H}{\partial t}=\operatorname{curl}[\mathbf{v} \times \mathbf{H}] .
\end{gathered}
$$

For this system, just as for the equations of hydrodynamics, the transition to canonical variables is accomplished using the Lagrange approach. For this we shall start from the well-known expression for the Lagrangian of a fluid interacting with the electromagnetic field in the MHD approximation. This means that in the Lagrangian we drop small terms of order $v / c$. Thus, for example, in the MHD approximation we should neglect the contribution from the electric field $(E \sim(v / c) H)$ compared to the corresponding contribution from the magnetic field. 
We also pay attention to one important consequence of Eqs. (10.22) following to which the magnetic field is frozen in a plasma [72]. It corresponds to that the vector $\mathbf{H} / \rho$ moves together with the fluid particles. In other words, each magnetic field line is displaced together with the particles that are on it. This fact allows one to regard the magnetic field $\mathbf{H}$ and the density $\rho$ as entering as generalized coordinates.

Thus the Lagrangian in the MHD approximation and including the constraint has the form:

$L=\int\left[\frac{\rho \mathbf{v}^{2}}{2}-\varepsilon(\rho)-\frac{\mathbf{H}^{2}}{8 \pi}+\mathbf{S}\left(\frac{\partial \mathbf{H}}{\partial t}-\operatorname{curl}[\mathbf{v} \times \mathbf{H}]\right)+\varphi\left(\frac{\partial \rho}{\partial t}+\operatorname{div} \rho \mathbf{v}\right)+\psi \operatorname{div} \mathbf{H}\right] d \mathbf{r}$.

Varying $L$ with respect to the variables $\mathbf{v}, \rho$ and $\mathbf{H}$, we get

$$
\begin{gathered}
\rho \mathbf{v}=[\mathbf{H} \times \operatorname{curl} \mathbf{S}]+\rho \nabla \varphi, \\
\frac{\partial \varphi}{\partial t}+\mathbf{v} \nabla \varphi-\frac{\mathbf{v}^{2}}{2}+\omega(\rho)=0, \\
\frac{\partial \mathbf{S}}{\partial t}+\frac{\mathbf{H}}{4 \pi}-[\mathbf{v} \times \text { curlS }]+\nabla \psi=0 .
\end{gathered}
$$

From this we see that the undetermined Lagrange multipliers enter as generalized momenta. The appropriate transition to these variables is accomplished using formula (10.23), and their evolution is determined from Eqs.(10.24), (10.25). The gauge function $\psi$ that enters these equations is chosen for convenience. For the natural condition, $\operatorname{div} \mathbf{S}=0$

$$
\psi=\Delta^{-1} \operatorname{div}[\mathbf{v} \times \operatorname{curl} \mathbf{S}]+\psi_{0}
$$

where $\psi_{0}$ is an arbitrary solution of the Laplace equation $\Delta \psi_{0}=0$. In particular, for finite motions of the plasma in a magnetic field $\mathbf{H}_{0}$, it is convenient to choose the quantity $\mathbf{S}$ so that $\mathbf{S} \rightarrow 0$ for $r \rightarrow \infty$. It is then obvious that

$$
\psi_{0}=\left(\mathbf{H}_{0} \mathbf{r}\right) / 4 \pi
$$

The equivalence of the system of equations here obtained and the MHD equations is verified by a direct substitution of the velocity in the equation of motion (10.22).

Now changing to the Hamiltonian description, we get [33]:

$$
\frac{\partial \rho}{\partial t}=\frac{\delta H}{\delta \varphi}, \frac{\partial \varphi}{\partial t}=-\frac{\delta H}{\delta \rho}, \frac{\partial \mathbf{H}}{\partial t}=\frac{\delta H}{\delta \mathbf{S}}, \frac{\partial \mathbf{S}}{\partial t}=-\frac{\delta H}{\delta \mathbf{H}},
$$

where the Hamiltonian

$$
H=\int\left[\frac{\rho \mathbf{v}^{2}}{2}+\varepsilon(\rho)+\frac{\mathbf{H}^{2}}{8 \pi}-\psi \operatorname{div} \mathbf{H}\right] d \mathbf{r}
$$

has a value that also coincides in value with the total energy of the system. 
Another way for introducing canonical variables in MHD was suggested in [73]. In this paper both the velocity and the magnetic field are parameterized in terms of the Clebsch-type potentials,

$$
\begin{gathered}
\mathbf{v}=\nabla \phi+\rho^{-1}(\mu \nabla \lambda+M \nabla \Lambda), \\
\mathbf{H}=[\nabla \lambda \times \nabla \Lambda] .
\end{gathered}
$$

So doing the variables $\lambda$ and $\mu, \Lambda$ and $M, \rho$ and $\phi$ form pairs of canonically conjugated values. It is possible to show that the given parametrization for $\mathbf{H}$ and $\mathbf{v}$ can be reduced by appropriate gauge choice to the change (10.23).

For the incompressible fluid the canonical variables are $\mathbf{H}$ and $\mathbf{S}$ : when taking into account that the potential $\varphi$ can be eliminated using the continuity equation

$$
\Delta \varphi=-\operatorname{div} \frac{1}{\rho_{0}}[\mathbf{H} \times \operatorname{curl} \mathbf{S}]
$$

the Hamiltonian simplifies into the form

$$
H=\int\left[\frac{\rho_{0} \mathbf{v}}{2}+\frac{\mathbf{H}^{2}}{8 \pi}+\psi \operatorname{div} \mathbf{H}\right] d \mathbf{r} .
$$

For barotropic flows the variables $(\rho, \phi) \quad(\mathbf{H}, \mathbf{S})$ determine the canonical Poisson bracket:

$$
\{F, G\}=\int\left\{\left[\frac{\delta F}{\delta \rho} \frac{\delta G}{\delta \varphi}-\frac{\delta F}{\delta \varphi} \frac{\delta G}{\delta \rho}\right]=\left[\frac{\delta F}{\delta \mathbf{H}} \frac{\delta G}{\delta \mathbf{S}}-\frac{\delta F}{\delta \mathbf{S}} \frac{\delta G}{\delta \mathbf{H}}\right]\right\} d \mathbf{r} .
$$

This bracket, as in the hydrodynamic case, allows recalculating to the natural variables, i.e., to the velocity $\mathbf{v}$, the density $\rho$ and the magnetic field $\mathbf{H}$. As a result, the noncanonical bracket represents itself the combination of (5.6) and the additional term containing the variational derivatives from the magnetic field [15]:

$$
\begin{gathered}
\{F, G\}=\int\left\{\left(\nabla \frac{\delta F}{\delta \rho}, \frac{\delta G}{\delta \mathbf{v}}\right)-\left(\nabla \frac{\delta G}{\delta \rho}, \frac{\delta F}{\delta \mathbf{v}}\right)\right\} d \mathbf{r} \\
+\int\left(\frac{\operatorname{rot} \mathbf{v}}{\rho},\left[\frac{\delta F}{\delta \mathbf{v}} \times \frac{\delta G}{\delta \mathbf{v}}\right]\right) d \mathbf{r}+\int\left(\frac{\mathbf{H}}{\rho},\left[\operatorname{rot} \frac{\delta F}{\delta \mathbf{H}} \times \frac{\delta G}{\delta \mathbf{v}}\right]\left[\operatorname{rot} \frac{\delta G}{\delta \mathbf{H}} \times \frac{\delta F}{\delta \mathbf{v}}\right]\right) d \mathbf{r} .
\end{gathered}
$$

Refusing from the barotropicy to this bracket one can get the additional term (compare with [15]):

$$
\int\left(\frac{\nabla S}{\rho},\left[\frac{\delta F}{\delta \mathbf{v}} \frac{\delta G}{\delta S}-\frac{\delta G}{\delta \mathbf{v}} \frac{\delta F}{\delta S}\right]\right) d \mathbf{r}
$$

The bracket $(10.27,10.28)$ occurs, as in a pure hydrodynamic limit $(\mathbf{H}=0)$, degenerate. 
The simplest annulators of the bracket was probably found in the paper [74]:

$$
C=\int \rho f\left(S, \frac{\mathbf{H} \nabla}{\rho} S,\left(\frac{\mathbf{H} \nabla}{\rho}\right)^{2} S, \ldots\right) d \mathbf{r}
$$

It can be verified by the direct calculations that the Lagrangian invariants which generate the integral (10.29) are written in the form:

$$
I_{n}=\left(\frac{\mathbf{H} \nabla}{\rho}\right)^{n} S .
$$

The integrals (10.29), however, are only one of the possible sets of the Eulerian integrals of motion. There exist the recurrent formula for construction of such type integrals they can be obtained from the Lagrangian invariants $I$, the frozen field $\mathbf{B}$, the density $\rho$ and the field of the Lamb impulse $\mathbf{p}[76,75]$. These quantities are defined from the corresponding equations of motion:

$$
\begin{gathered}
\frac{\partial I}{\partial t}+(\mathbf{v} \nabla) I=0 \\
\frac{\partial \mathbf{B}}{\partial t}+(\mathbf{v} \nabla) \mathbf{B}=(\mathbf{B} \nabla) \mathbf{v}, \\
\frac{\partial \mathbf{p}}{\partial t}+(\mathbf{v} \nabla) \mathbf{p}+(\mathbf{p} \nabla) \mathbf{v}+[\mathbf{p} \times \operatorname{rot} \mathbf{v}]=0 .
\end{gathered}
$$

Recurrent procedure for the construction of Lagrange invariants consists of a few steps.

At first, it is easy to verify that the definition of the fields $\mathbf{B}$ and $\mathbf{p}$ by means of equations (10.32) and (10.33) remains without changes after their multiplication by $I$ :

$$
\mathbf{B}^{\prime}=I \mathbf{B}, \quad \mathbf{p}^{\prime}=I \mathbf{p},
$$

i.e. new fields $\mathbf{B}^{\prime} \mathbf{p}^{\prime}$ obey the same equations as those for $\mathbf{B}$ and $\mathbf{p}$.

At the next step by the given $I, \mathbf{p}, \mathbf{B}$ we construct a new set of quantities $I^{\prime}, \mathbf{p}^{\prime}, \mathbf{B}^{\prime}$, which possess the same properties:

$$
\mathbf{p}^{\prime}=\nabla I, \mathbf{B}^{\prime}=\frac{1}{\rho} \operatorname{rot} \mathbf{p}^{\prime}, I^{\prime}=\frac{1}{\rho} \operatorname{div}(\rho \mathbf{B}) .
$$

At the third step, by substituting (10.34) into (10.35), we obtain

$$
I^{\prime}=\mathbf{v} \cdot \mathbf{B}, \mathbf{B}^{\prime}=\frac{1}{\rho}\left[\mathbf{p}, \mathbf{p}^{\prime}\right], \mathbf{B}^{\prime}=\frac{1}{\rho}\left[\nabla I, \nabla I^{\prime}\right]
$$

The further recursion gives the relations:

$$
\mathbf{p}=\rho\left[\mathbf{B} \times \mathbf{B}^{\prime}\right], I=\frac{1}{\rho}\left(\mathbf{p},\left[\nabla I^{\prime} \nabla I^{\prime \prime}\right]\right), I=\frac{1}{\rho}\left(\nabla I^{\prime}\left[\nabla I^{\prime \prime} \times \nabla I^{\prime \prime \prime}\right]\right) .
$$


Because an arbitrary function of Lagrangian variables is again a Lagrangian invariant then this in combination with $(10.34),(10.35,(10.36),(10.37)$ set the prescription of the Lagrangian invariant reproduction. For example, the Lagrangian invariants of the first generation, consisting of the quantities $\rho, \mathbf{p}, \mathbf{B}$ and three Lagrangian invariants given initially plus a lack constructed by means of (10.34)-(10.37), can be represented in the form [75]:

$$
\begin{gathered}
I_{0}^{\prime}=\mathbf{p} \times \mathbf{B}, \quad I_{i k}^{\prime}=\frac{1}{\rho}\left(\mathbf{p}\left[\nabla I_{i} \nabla I_{k}\right]\right), \\
I_{k}^{\prime}=(\mathbf{B} \nabla) I_{k}, \quad I^{\prime}=\frac{1}{\rho}\left(\nabla I_{1}\left[\nabla I_{2} \nabla I_{3}\right]\right) .
\end{gathered}
$$

Using this procedure of multiplication one can get the next generations of Lagrangian invariants.

Of particular interest is a question about Lagrangian invariant construction in two-dimensional case. Here to construct an infinite hierarchy of invariants it is enough to have two input invariants. As it was shown in [78] in 2D case the whole set of conserved quantities forms highly complicated Lie algebra, which contains very interesting subalgebra, i.e., the loop algebra with layer in algebra conserving area of diffeomorphisms of a plane.

Let us apply this approach to the MHD equations.

In the MHD case with an arbitrary equation of state $p=p(\rho, S)$ one should take in the capacity of $I$ one should consider the entropy $S$, instead of the vector field $\mathbf{B}$ - $\mathbf{H} / \rho$, and the field $\mathbf{p}$ should be changed by the vector potential of the magnetic field $\mathbf{A}(\mathbf{H}=\operatorname{rot} \mathbf{A})$, imposing the gauge [75]:

$$
\frac{\partial \mathbf{A}}{\partial t}=[\mathbf{v} \times \operatorname{rot} \mathbf{A}]-\nabla(\mathbf{v A}) \equiv-(\mathbf{v} \nabla) \mathbf{A}-(\mathbf{A} \nabla) \mathbf{v}-[\operatorname{rot} \mathbf{v} \times \mathbf{A}] .
$$

It is easy to see that for MHD the transformation (10.35) reads as follows:

$$
\mathbf{A}^{\prime}=\nabla S, \quad \mathbf{B}^{\prime}=\frac{\mathbf{H}}{\rho}, \quad I^{\prime}=0 .
$$

The first equation reflects the gauge freedom of the vector potential, the second formula in this case is the definition of the frozen field.

If now in the third formula of (10.35) one takes instead of $\mathbf{B}$ its transforming value from (10.34), then as a result one can get the second (after $S$ ) Lagrangian invariant:

$$
I_{2}=\frac{(\mathbf{H} \nabla) S}{\rho} .
$$

By its structure it is familiar to the Ertel invariant for hydrodynamic flows. Multiple using of this transformation leads to the invariants (10.30):

$$
I_{n}=\left(\frac{H \nabla}{\rho}\right)^{n} S \text {. }
$$


The transformation (10.36) generates another Lagrangian invariant

$$
I_{3}=\frac{(\mathbf{A}, \mathbf{H})}{\rho} .
$$

Integration of $I_{3}$ with the help of formula (6.1) reduces to the integral of motion

$$
I_{k}=\int(\mathbf{A}, \mathbf{H}) d \mathbf{r}
$$

which characterizes the degree of knottiness of lines of the magnetic field $\mathbf{H}$ [56].

Representation of three invariants $I_{1}=S, I_{2}$ and $I_{3}$, and also of the magnetic field $\mathbf{H}$ and its vector potential A permits, by use of the formula (10.38), to find all sets of Lagrangian invariants and together with them the Eulerian integrals of motion

$$
C=\int \rho f\left(I_{1}, I_{2}, \ldots\right) d \mathbf{r} .
$$

In the case of barotropic flows the given recursion undergoes changes. At first, one should exclude the entropy $S$ as a quantity not entering into the equations of motion. Therefore from the set of Lagrangian invariants $I_{i}(i=1,2,3)$ of the first generation, explicitly expressed in terms of $\mathbf{H}$ and $\rho$, the only invariant $I_{2}=\frac{\mathbf{A H}}{\rho}$ remains. With its help all series of the integrals of motion is written as follows [49], $[75]$ :

$$
C=\int \rho f\left(I_{2}, \frac{H \nabla}{\rho} I_{2}, \ldots\right) d \mathbf{r}
$$

Besides, in the barotropic case a new integral should be added. This is the topological invariant

$$
C_{t}=\int(\mathbf{v}, \mathbf{H}) d \mathbf{r}
$$

characterizing the degree of cross knottiness of the magnetic field and velocity lines $[56]$.

It is possible to show that all presented above integrals are the Casimirs relative to the bracket $(10.27,10.28)$.

Thus, we demostrated how canonical variables are introduced for the hydrodynamical models of plasma. These variables to some extent generalize the Clebsch variables for ideal hydrodynamics. Their difference exhibits in that, firstly, a number of canonical variables increases, so that electromagnetic field itself represents additional canonical variable, and, secondly, due to this fact the Hamiltonian structure of the equations changes (complicates) that becomes especially transparent for MHD.

\section{The Hamiltonian Formalism in Kinetics}

In this section we introduce a Hamiltonian structure into the collisionless kinetic equation of the self-consistent type. We consider the simplest example that has 
sufficient contents from the point of view of generalization: the Vlasov kinetic equation for the distribution function $f$ describing potential (electric field $E=-\nabla \varphi$ ) oscillations of electrons relative to a homogeneous background of ions with density $n_{0}$ :

$$
\begin{gathered}
\frac{\partial f}{\partial t}+(\mathbf{v} \nabla) f-\nabla \varphi \frac{\partial f}{\partial \mathbf{v}}=0 \\
\Delta \varphi=-4 \pi\left[\int f d \mathbf{v}-n_{0}\right] \quad(e=m=1) .
\end{gathered}
$$

Kinetic equations of this type also should be included with systems of hydrodynamic type. In the phase space (r, v) Eq.(11.1) describes the motion of an incompressible "fluid", whose density is convected together with the "fluid". The behavior of the system here is in many ways similar to the situation which holds in a stratified fluid. In order to transform to canonical coordinates, we introduce the Lagrange coordinate $\xi$, which we determine from the condition that the distribution function $f$ be equal to the equilibrium distribution function $f_{0}(\xi)$, not necessarily Maxwellian:

$$
f(\mathbf{r}, \mathbf{v}, t)=f_{0}(\xi) \text { or } \mathbf{v}=\mathbf{V}(\mathbf{r}, \xi, t) .
$$

Such a representation can be expressed in the integral form:

$$
f(\mathbf{r}, \mathbf{v}, t)=\int F(\mathbf{r}, \xi, t) \delta(\mathbf{v}-\mathbf{V}(\mathbf{r}, \xi, t)) d \xi
$$

Substitution of (11.2) in (11.1) leads to the system of equations

$$
\begin{gathered}
\frac{\partial F}{\partial t}+\operatorname{div}(F \mathbf{V})=0, \\
\frac{\partial \mathbf{V}}{\partial t}+(\mathbf{V} \nabla) \mathbf{V}=-\nabla \varphi \\
\Delta \varphi=-4 \pi\left[\int F(\xi, \mathbf{r}, t) d \xi-n_{0}\right] .
\end{gathered}
$$

The internal energy of this system is a functional of the "density" $F$ :

$$
\begin{gathered}
\mathcal{E}_{i n}=\frac{1}{8 \pi} \int(\nabla \varphi)^{2} d \mathbf{r} \\
=\frac{1}{2} \int \frac{\left[\int F(\xi, \mathbf{r}) d \xi-n_{0}\right]\left[\int F\left(\xi^{\prime}, \mathbf{r}^{\prime}\right) d \xi^{\prime}-n_{0}\right]}{\left|\mathbf{r}-\mathbf{r}^{\prime}\right|} d \mathbf{r} d \mathbf{r}^{\prime}
\end{gathered}
$$

and therefore, according to the classification of Section 4 belongs to the type of (4.1), $(4.2)$.

Canonical variables for (11.3)-(11.5) are introduced in the standard way. For potential "flows" $\mathbf{V}=\nabla \Phi$ the equations of motion have the form of the Hamiltonian equations

$$
\frac{\partial \Phi}{\partial t}=-\frac{\delta H}{\delta F}, \frac{\partial F}{\partial t}=\frac{\delta H}{\delta \Phi}
$$


where

$$
H=\int \frac{F \mathbf{v}^{2}}{2} d \xi d \mathbf{r}+\mathcal{E}_{i n}
$$

i.e., the Poisson bracket has the canonical form:

$$
\{S, T\}=\int d \xi d \mathbf{r}\left[\frac{\delta S}{\delta F} \frac{\delta T}{\delta \Phi}-\frac{\delta T}{\delta F} \frac{\delta S}{\delta \Phi}\right] .
$$

It can be expressed in terms of the distribution function $f$. By using (11.2), together with simple transformations, one can get a bracket which was first obtained in [16]:

$$
\{S, T\}=\int f\left[\left(\frac{\partial}{\partial \mathbf{v}} \frac{\delta S}{\delta f}\right)\left(\frac{\partial}{\partial \mathbf{r}} \frac{\delta T}{\delta f}\right)-\left(\frac{\partial}{\partial \mathbf{r}} \frac{\delta S}{\delta f}\right)\left(\frac{\partial}{\partial \mathbf{v}} \frac{\delta T}{\delta f}\right)\right] d \mathbf{r} d \mathbf{v} .
$$

Canonical variables are introduced analogously in the Vlasov-Maxwell equations, where the canonical Poisson bracket allows for change to the bracket [16], locally dependent on the distribution function and the electromagnetic field.

In concluding this section we mention similar another important example, in which there is an analogous construction. This is the Benney equations, describing surface waves in the approximation of "shallow" water, where the flow of the fluid is not assumed to be potential:

$$
\begin{gathered}
h_{1}+\operatorname{div} \int_{0}^{h} \mathbf{U} d z=0, \\
U_{1}+(\mathbf{U} \nabla) \mathbf{U}+W \frac{\partial \mathbf{U}}{\partial z}+\nabla h=0 \\
\frac{\partial W}{\partial z}+\operatorname{div} \mathbf{U}=0 .
\end{gathered}
$$

Here $h=h(\mathbf{r}, t)(\mathbf{r}=(x, y), 0<z<h)$ is the boundary of the free surface of the fluid, $\mathbf{U}=\mathbf{U}(\mathbf{r}, z)$ is the horizontal velocity vector, $W=W(\mathbf{r}, z)$ is the vertical component of the velocity, and $g=1$. We first show that the system (11.6)-(11.8) can be reduced to an infinite system of two-dimensional hydrodynamic equations.

We introduce a coordinate $\xi(0<\xi<l)$ which enumerates each layer of the fluid in equilibrium along the direction $z$. Then the coordinate of each layer at time $t$ will be given by the functions:

$$
z=z(\mathbf{r}, \xi, t), h(\mathbf{r}, t)=z(\mathbf{r}, l, t) .
$$

It is clear that the equations examined in terms of this function are similar to (9.4) with

$$
\frac{d z}{d t}=\frac{\partial z}{\partial t}+(\mathbf{U} \nabla) z=W
$$


Setting $\xi=l$ we see that (11.6) follows from (11.9). Derivatives taken for constant $\xi$ and $z$ are linked by the formulas:

$$
\left(\frac{\partial}{\partial t}\right)_{z}=\left(\frac{\partial}{\partial t}\right)_{\xi}-\frac{z_{t}}{\eta} \frac{\partial}{\partial \xi}, \quad(\nabla)_{z}=(\nabla)_{\xi}-\frac{\nabla z}{\eta} \frac{\partial}{\partial \xi} .
$$

In addition, we dispose of:

$$
\frac{\partial}{\partial z}=\frac{1}{\eta} \frac{\partial}{\partial \xi}
$$

where

$$
\eta(\mathbf{r}, \xi, t)=\frac{\partial z}{\partial \xi}
$$

Differentiating the relation (11.9) with respect to $\xi$ and using formulas (11.10)(11.11), we easily obtain the equation

$$
\frac{\partial \eta}{\partial t}+\operatorname{div}(\eta \mathbf{U})=0
$$

(Here and everywhere below the derivatives are taken at constant $\xi$ ).

Applying the same formulas to (11.7), we find, after transformations,

$$
\frac{\partial \mathbf{U}}{\partial t}+(\mathbf{U} \nabla) \mathbf{U}+\nabla h=0
$$

where $h$ and $\eta$ are connected by the relation

$$
h=\int_{0}^{l} \eta(\mathbf{r}, \xi, t) d \xi .
$$

The system (11.12)-(11.14) is similar to that considered above and differs from it only in the consistency condition (11.14). Therefore, the canonical variables for potential (in the $x, y$ plane) flows $(\mathbf{U}=\nabla \varphi)$ remain the same [30]:

$$
\frac{\partial \varphi}{\partial t}=-\frac{\delta H}{\delta \eta}, \frac{\partial \eta}{\partial t}=\frac{\delta H}{\delta \varphi}
$$

where

$$
H=\frac{1}{2} \int d \xi d \mathbf{r} \eta(\nabla \varphi)^{2}+\frac{1}{2} \int d \mathbf{r} h^{2}
$$

If the flow depends only on $x$, the Hamiltonian structure can be given in terms of the variables $\eta$ and $U$ :

$$
\frac{\partial \eta}{\partial t}=-\frac{\partial}{\partial x} \frac{\delta H}{\delta U}, \frac{\partial U}{\partial t}=-\frac{\partial}{\partial x} \frac{\delta H}{\delta \eta}
$$


It should be added that for one-dimensional flows another method for introducing a Hamiltonian structure was developed in paper [32]. One can show that the Hamiltonian structure introduced in [32] is equivalent to the structure (11.15).

At the end of this section we would like to pay attention to one more paper [79] where, in fact, the same idea as for the Vlasov and Benney equations was used. In [79] Virasoro supposes to describe flows of stratified fluid by use of mixed, LagrangianEulerian representation. For two-dimensional flows independent variables serve the horizontal coordinate $x$ and the Lagrangian coordinate, $\beta$, labeled the density levels. In the case of two-dimensional hydrodynamics, when also the suggested scheme can be applied, one of the coordinates (Lagrangian) labels the vorticity levels and another coordinate may be, for example, Cartesian $x$. Virasoro, from the very beginning, comes from the variational principle in the Lagrange form (7.6), and then performs a transformation to new variables, by introducing the generating function of this transformation. Just this function in the Lagrangian plays a role of the generated coordinate.

Approximately the same ideas lie in the base of papers [38, 39] where for the equation (5.11) describing the Rossby waves the Gardner-Zakharov-Faddeev brackets is derived from noncanonical Poisson brackets.

\section{Classical Perturbation Theory and Reduction of Hamiltonians}

If in the previous sections we dealt with introducing the Hamiltonian structure, then further we will suppose that we was able by some way to introduce canonical variables and together with them normal variables diagonalizing a quadratic part of Hamiltonian. In this section we turn to the classical perturbation theory for the wave Hamiltonian systems which is based on an assumption about smallness of wave amplitudes. Difference of the wave systems from the finite-dimensional ones consists in that application of the perturbation theory to the wave systems leads to appearance of the resonant dominators not at separate points, as it is for finite-dimensional equations, but on the whole manifolds. By their classification, we arrive at the whole set of standard Hamiltonians and corresponding equations. In particular, many well-known equations such that the nonlinear Schrodinger equation, the KDV equation, the KP equation, etc. are among this set.

Suppose that in a medium there is one type of wave with dispersion law $\omega(k)$ and amplitudes $a(k)$, whose evolution is determined be Eq.(3.7):

$$
\frac{\partial a_{k}}{\partial t}=-i \frac{\delta H}{\delta a_{k}^{*}}
$$

where

$$
H=H_{0}+H_{1}+\ldots
$$




$$
\begin{gathered}
H_{0}=\int \omega_{k}\left|a_{k}\right|^{2} d k \\
H_{1}=\int\left(V_{k k_{1} k_{2}} a_{k}^{*} a_{k_{1}} a_{k_{2}}+\text { c.c. }\right) \delta_{k-k_{1}-k_{2}} d \mathbf{k} d \mathbf{k}_{1} d \mathbf{k}_{2} \\
+\frac{1}{3} \int\left(U_{k k_{1} k_{2}} a_{k}^{*} a_{k_{2}}^{*} a_{k_{2}}^{*}+\text { c.c. }\right) \delta_{k+k_{1}+k_{2}} d \mathbf{k} d \mathbf{k}_{1} d \mathbf{k}_{2} .
\end{gathered}
$$

Consider a transformation from the variables $a(k)$ to new variables $c(k)$ in the form of integral power series,

$$
\begin{array}{r}
a_{k}=c_{k}+\int L_{k k_{1} k_{2}} c_{k_{1}} c_{k_{2}} \delta_{k-k_{1}-k_{2}} d \mathbf{k}_{1} d \mathbf{k}_{2} \\
+\int M_{k k_{1} k_{2}} c_{k} c_{k_{2}}^{*} \delta\left(k_{2}-k-k_{1}\right) d \mathbf{k}_{1} d \mathbf{k}_{2} \\
\left.\quad+\int N_{k k_{1} k_{2}} c_{k}^{*} c_{k_{1}}^{*} \delta_{k+k_{1}+k_{2}} d \mathbf{k}_{1}\right) d \mathbf{k}_{2}+\ldots
\end{array}
$$

We require such a transformation to eliminate the third order terms from the Hamiltonian and that it must be canonical. The last item means that

$$
\left\{c_{k}, c_{k^{\prime}}^{*}\right\}=\delta_{k-k^{\prime}}, \quad\left\{c_{k}, c_{k^{\prime}}\right\}=\left\{c_{k}^{*}, c_{k^{\prime}}^{*}\right\}=0 .
$$

From these two requirements, after simple algebra, we can find that

$$
\begin{aligned}
a_{k}= & c_{k}-\int \frac{V_{k k_{1} k_{2}} c_{k_{1}} c_{k_{2}}}{\omega_{k}-\omega_{k_{1}}-\omega_{k_{2}}} \delta_{k-k_{1}-k_{2}} d \mathbf{k}_{1} d \mathbf{k}_{2} \\
& +2 \int \frac{V_{k_{2} k k_{1}}^{*} c_{k} c_{k_{2}}^{*}}{\omega_{k_{2}}-\omega_{k}-\omega_{k_{1}}} \delta_{k_{2}-k-k_{1}} d \mathbf{k}_{1} d \mathbf{k}_{2} \\
- & \int \frac{U_{k k k_{2}} c_{k}^{*} c_{k_{1}}^{*}}{\omega_{k}+\omega_{k_{1}}+\omega_{k_{2}}} \delta_{k+k_{1}+k_{2}} d \mathbf{k}_{1} d \mathbf{k}_{2}+\ldots
\end{aligned}
$$

Here the first two integral terms guarantee the cancelation in $H$ (3.8) of the second two terms, while the last term gives the cancelation of the other two ones, proportional to $a^{*} a^{*} a^{*}$ and $a a a$. These two transformations (elimination of both pairs from $H$ ) are independent and can be carried out separately. This procedure for successive elimination of perturbation terms in the Hamiltonian expansion by means of canonical transformations is called classical perturbation theory. In constructing such a theory we quickly come up against the problem of "small denominators", related in the present case to the appearance of nonintegrable singularities near the manifolds

$$
\begin{gathered}
\omega_{k}+\ldots+\omega_{k_{i}}-\omega_{k_{i+1}}-\ldots-\omega_{k_{n}}=0 \\
\mathbf{k}+\ldots+\mathbf{k}_{i}-\mathbf{k}_{i+1}-\ldots-\mathbf{k}_{n}=0
\end{gathered}
$$

which give the condition for an $n$th order resonance. The simplest manifolds already appear in the elimination of the three-wave Hamiltonian (3.8), when (cf. (12.5)): 


$$
\begin{gathered}
\omega_{k}+\omega_{k_{1}}+\omega_{k_{2}}=0 \\
\mathbf{k}+\mathbf{k}_{1}+\mathbf{k}_{2}=0
\end{gathered}
$$

and

$$
\begin{gathered}
\omega_{k}-\omega_{k_{1}}-\omega_{k_{2}}=0 \\
\mathbf{k}-\mathbf{k}_{1}-\mathbf{k}_{2}=0
\end{gathered}
$$

Satisfying the first condition is possible if waves with negative energy exist in the medium, and then one of the frequencies $\omega_{k}$ must be negative. Such a situation, as a rule, occurs in unstable media, for example, in a plasma with a current. If there are no waves with negative energy in the medium, then the terms proportional to $a^{*} a^{*} a^{*}$ and $a a a$ can be eliminated from $H_{1}$ by a canonical transformation, and in this sense they are unimportant (non-resonant).

The possible existence of solutions of the system (12.7) depends on the form of the functions $\omega(\mathbf{k})$. For isotropic media, in which $\omega(k)$ depends only on $|\mathbf{k}|$, there is no solution if $\omega(0)=0$ and $\omega^{\prime \prime}(k)<0$. Such a situation is realized, for example, for surface gravitational waves. For capillary waves the resonance conditions (12.7) are satisfied.

If the conditions (12.6), (12.7) have no solutions then the three-wave terms are eliminated. Among the fourth order terms the important one is the Hamiltonian in the form

$$
H_{3}=\int T_{k_{1} k_{2} k_{3} k_{4}} a_{k_{1}}^{*} a_{k_{2}}^{*} a_{k_{3}} a_{k_{4}} \delta_{k_{1}+k_{2}-k_{3}-k_{4}} \Pi d \mathbf{k}_{i}
$$

for which the resonance condition

$$
\begin{gathered}
\omega_{k_{1}}+\omega_{k_{2}}-\omega_{k_{3}}-\omega_{k_{4}}=0, \\
\mathbf{k}_{1}+\mathbf{k}_{2}-\mathbf{k}_{3}-\mathbf{k}_{4}=0
\end{gathered}
$$

has solutions independent of the form of $\omega(k)$. Here the three-wave interaction leads to a re-normalization of the vertex $T_{k k_{1} k_{2} k_{3}}$ in (12.8) (see, [85]):

$$
\begin{array}{r}
T_{k k_{1} k_{2} k_{3}}=T_{k k_{1} k_{2} k_{3}}^{(0)}-2 \frac{U_{-k_{2}-k_{3}, k_{2} k_{3}} U_{-k-k_{1}, k k_{1}}^{*}}{\omega_{k+k_{1}}+\omega_{k}+\omega_{k_{1}}} \\
+2 \frac{V_{k_{2}+k_{3} k_{2} k_{3} V_{k+k_{1} k k_{1}}^{*}}}{\omega_{k+k_{1}}-\omega_{k}-\omega_{k_{1}}}-2 \frac{V_{k k_{2} k-k_{2}} V_{k_{3} k_{1} k_{3}-k_{1}}^{*}}{\omega_{k_{3}-k_{1}}+\omega_{k_{1}}-\omega_{k_{3}}} \\
-2 \frac{V_{k_{1} k_{3} k_{1}-k_{3}} V_{k_{2} k k_{2}-k}^{*}}{\omega_{k_{2}-k}+\omega_{k}-\omega_{k_{2}}}-2 \frac{V_{k_{1} k_{2} k_{1}-k_{2}} V_{k_{3}}^{*}-k k_{3}-k}{\omega_{k_{3}-k}+\omega_{k}-\omega_{k_{3}}} \\
-2 \frac{V_{k k_{3} k-k_{3}} V_{k_{2} k_{1} k_{2}-k_{1}}^{*}}{\omega_{k_{2}-k}+\omega_{k_{1}}-\omega_{k_{2}}} .
\end{array}
$$


Thus, we arrive at a sequence of standard interaction Hamiltonians:

$$
H_{d}=\int\left(V_{k k_{1} k_{2}} a_{k}^{*} a_{k_{1}} a_{k_{2}}+\text { c.c. }\right) \delta_{k-k_{1}-k_{2}} d \mathbf{k} d \mathbf{k}_{1} d \mathbf{k}_{2}
$$

responsible for the process of decay $1 \rightarrow 2$ and the inverse process of fusion $2 \rightarrow 1$; the Hamiltonian

$$
H_{e x}=\frac{1}{3} \int\left(U_{k k_{1} k_{2}}^{*} a_{k}^{*} a_{k_{1}}^{*} a_{k_{2}}^{*}+\text { c.c. }\right) \delta_{k+k_{1}+k_{2}} d \mathbf{k} d \mathbf{k}_{1} d \mathbf{k}_{2},
$$

describing the so-called explosive instability, in which three quanta of the wave field are created simultaneously from the vacuum $(0 \rightarrow 3)$, the Hamiltonian

$$
H_{s c}=\int T_{k k_{1} k_{2} k_{3}} a_{k}^{*} a_{k_{1}}^{*} a_{k_{2}} a_{k_{3}} \delta_{k+k_{1}-k_{2}-k_{3}} \Pi d \mathbf{k}_{i}
$$

responsible for the process $2 \rightarrow 2$, etc.

If several types of waves exist in the medium, the list of standard Hamiltonians is greatly increased. We give one of them, responsible for the interaction of highfrequency and low-frequency waves,

$$
H_{\text {int }}=\int\left(V_{k k_{1} k_{2}} b_{k} a_{k_{1}}^{*} a_{k_{2}}+\text { c.c. }\right) \delta_{k-k_{1}-k_{2}} d \mathbf{k} d \mathbf{k}_{1} d \mathbf{k}_{2} .
$$

A Hamiltonian of type (12.13) describes the interaction of light and sound in dielectrics, Langmuir and ion-acoustic waves in plasma, etc.

In describing a system of nonlinear waves by means of some standard interaction Hamiltonian, we are naturally assuming that the level of nonlinearity, characterized by the wave amplitude, is small. Despite these limitations, the resulting phenomena are quite rich. Many of them can already be understood starting from the simplest models that arise from the reduction of the standard Hamiltonians.

As a first example let us consider the interaction of three spectrally narrow wave packets with wave vectors lying near $\mathbf{k}_{1}, \mathbf{k}_{2}$ and $\mathbf{k}_{3}$. Such an interaction is resonant if, for instance,

$$
\begin{gathered}
\omega\left(\mathbf{k}_{1}\right)=\omega\left(\mathbf{k}_{2}\right)+\omega\left(\mathbf{k}_{3}\right) \\
\mathbf{k}_{1}=\mathbf{k}_{2}+\mathbf{k}_{3} .
\end{gathered}
$$

For this case $a(k)$ is representative in the form

$$
a(\mathbf{k})=a_{1}(\mathbf{k})+a_{2}(\mathbf{k})+a_{3}(\mathbf{k})
$$

where $a_{1}, a_{2}, a_{3}$ are the wave amplitudes of the packets concentrated near points $\mathbf{k}=\mathbf{k}_{i}(i=1,2,3)$, the characteristic width $\kappa_{i}$ of the each packet is assumed to be small compared with $\left|\mathbf{k}_{i}\right|$. For such an interaction, a canonical transformation reduces $H_{d}(12.10)$ to the form:

$$
H_{\text {int }}=2 \int\left[V a_{1}^{*}\left(k_{1}\right) a_{2}\left(k_{2}\right) a_{3}\left(k_{3}\right)+\text { c.c. }\right] \delta_{k_{1}-k_{2}-k_{3}} \Pi d \mathbf{k}_{i} .
$$


Now using the narrowness of these packets, we set

$$
\omega\left(\mathbf{k}_{1}+\kappa\right)=\omega\left(\mathbf{k}_{i}\right)+\kappa \mathbf{v}_{i}, \mathbf{v}_{i}=\partial \omega / \partial \mathbf{k}_{i}
$$

in $H_{0}$ and make the change of variables:

$$
c_{i}(\kappa)=a_{i}(\mathbf{k}) e^{i \omega\left(k_{i}\right) t}\left(\kappa=\mathbf{k}-\mathbf{k}_{\mathbf{i}}\right) .
$$

As a result,

$$
H \rightarrow H-\sum \int \omega_{i}\left|c_{i}\right|^{2} d \kappa
$$

Taking the inverse Fourier transform of this Hamiltonian, using the formula

$$
\psi_{i}(x)=\frac{1}{(2 \pi)^{3 / 2}} \int c_{i}(\kappa) e^{i \kappa \mathbf{r}} d \kappa
$$

we obtain the well-known equations for resonant interaction [80]:

$$
\begin{aligned}
\frac{\partial \psi_{1}}{\partial t}+\left(\mathbf{v}_{1} \nabla\right) \psi_{1} & =-\frac{i V}{(2 \pi)^{3 / 2}} \psi_{2} \psi_{3} \\
\frac{\partial \psi_{2}}{\partial t}+\left(\mathbf{v}_{2} \nabla\right) \psi_{2} & =-\frac{i V^{*}}{(2 \pi)^{3 / 2}} \psi_{1} \psi_{3}^{*} \\
\frac{\partial \psi_{3}}{\partial t}+\left(\mathbf{v}_{3} \nabla\right) \psi_{3} & =-\frac{i V^{*}}{(2 \pi)^{3 / 2}} \psi_{2} \psi_{3}^{*}
\end{aligned}
$$

In similar fashion one gets the system of equations for describing the explosive instability of three wave packets. In this case the interaction Hamiltonian for the packets arises as the result of reduction of the Hamiltonian (12.11):

$$
\begin{aligned}
\frac{\partial \psi_{1}}{\partial t}+\left(\mathbf{v}_{1} \nabla\right) \psi_{1} & =-\frac{i U^{*}}{(2 \pi)^{3 / 2}} \psi_{2}^{*} \psi_{3}^{*} \\
\frac{\partial \psi_{2}}{\partial t}+\left(\mathbf{v}_{2} \nabla\right) \psi_{2} & =-\frac{i U^{*}}{(2 \pi)^{3 / 2}} \psi_{1}^{*} \psi_{3}^{*} \\
\frac{\partial \psi_{3}}{\partial t}+\left(\mathbf{v}_{3} \nabla\right) \psi_{3} & =-\frac{i U^{*}}{(2 \pi)^{3 / 2}} \psi_{2}^{*} \psi_{3}^{*}
\end{aligned}
$$

The following example refers to the reduction of the Hamiltonian (12.12) for a single spectrally narrow wave packet. Suppose that the center of the packet is at $\mathbf{k}_{0}$ then setting

$$
\begin{gathered}
a(\mathbf{k})=c(\kappa) \exp \left(-i \omega_{k_{0}} t\right), \quad \mathbf{k}=\mathbf{k}_{\mathbf{0}}+\kappa \\
H \rightarrow H-\omega\left(k_{0}\right) \int|c(\kappa)|^{2} d \kappa
\end{gathered}
$$




$$
\omega(\mathbf{k})=\omega\left(\mathbf{k}_{\mathbf{0}}+\kappa\right)=\omega\left(\mathbf{k}_{\mathbf{0}}\right)+\kappa \mathbf{v}_{g}+\frac{1}{2} \frac{\partial^{2} \omega}{\partial k_{\alpha} \partial k_{\beta}} \kappa_{\alpha} \kappa_{\beta}
$$

we get for the envelope $\psi(\mathbf{r})$ the nonlinear Schrödinger equation (NLSE):

$$
i\left(\psi_{t}+\mathbf{v}_{g} \nabla \psi\right)+\frac{\omega_{\alpha \beta}}{2} \frac{\partial^{2} \psi}{\partial x_{\alpha} \partial x_{\beta}}+\frac{T}{(2 \pi)^{3}}|\psi|^{2} \psi=0
$$

where

$$
\omega_{\alpha \beta}=\frac{\partial^{2} \omega}{\partial k_{\alpha} \partial k_{\beta}} .
$$

Equation (12.20) describes the self-interaction of a spectrally narrow wave packet in a nonlinear medium. In an isotropic medium, when the tensor

$$
\omega_{\alpha \beta}=\frac{v_{g}}{2 k_{0}}\left(\delta_{\alpha \beta}-n_{\alpha} n_{\beta}\right)+\omega^{\prime \prime} n_{\alpha} n_{\beta} \quad\left(\mathbf{n}=\frac{\mathbf{k}}{k}\right)
$$

this equation simplifies to the form

$$
i\left(\psi_{t}+v_{g} \psi_{x}\right)+\frac{v_{g}}{2 k_{0}} \Delta_{\perp} \psi+\frac{\omega^{\prime \prime}}{2} \psi_{x x}+\frac{T}{(2 \pi)^{3}}|\psi|^{2} \psi=0,
$$

where the $x$ axis coincides with the direction of the group velocity. In this equation the second term is responsible for the propagation of the wave packet as a whole with the group velocity $v_{g}$ (this term can be evidently excluded by passing to the system of reference moving with $\mathbf{v}_{\mathbf{g}}$ ); the next term describes the diffraction of the packet in the plane transverse to $\mathbf{v}_{\mathbf{g}}$, the fourth term corresponds to the dispersion of the broadening along the $x$-direction, finally, the last term in (12.21) accounts for the nonlinearity.

After performing in this equation rescaling transformations, the NLSE (12.21) can be written in the following canonical form,

$$
i \psi_{t}+\Delta_{\perp} \psi+\sigma \psi_{x x}+\eta|\psi|^{2} \psi=0
$$

where $\sigma=\operatorname{sign}\left(\omega^{\prime \prime} v_{g}\right)$ and $\eta=\operatorname{sign}\left(T v_{g}\right)$. This equation can be considered as the Shrödinger equation for a quantum particle motion in self-consistent potential $U=-\eta|\psi|^{2}$ with a positive transverse mass and a longitudinal mass sing of which coincides with $\sigma$. This means that the character of interaction in transverse and longitudinal directions are different in dependence of signs of $\eta$ and $\sigma$. If $\eta>0$, then in transverse direction the attraction takes place and the packet has to be compressed due the nonlinear interaction. In the opposite case, $(\eta<0)$, the nonlinearity helps to the diffraction broadening. The same situation is with the longitudinal motion. If $\sigma \eta=1$, then the compression takes place along the group velocity direction and respectively the repulsion in the opposite case $(\sigma \eta=-1)$. There exists the only 
variant $\sigma=\eta=1$, when simultaneously the nonlinearity leads to the packet compression in all directions. Just in this case the wave collapse is possible (for review see $[86])$.

Thus, depending on $\sigma$ and $\eta$, there exist four canonical forms for the NLSE:

$$
\begin{gathered}
i \psi_{t}+\Delta \psi+|\psi|^{2} \psi=0 ; \\
i \psi_{t}+\Delta_{\perp} \psi-\psi_{x x}+|\psi|^{2} \psi=0 ; \\
i \psi_{t}+\Delta \psi-|\psi|^{2} \psi=0 ; \\
i \psi_{t}+\Delta_{\perp} \psi-\psi_{x x}-|\psi|^{2} \psi=0 .
\end{gathered}
$$

All these equations belong to the Hamiltonian type, they can be written as

$$
i \psi_{t}=\frac{\delta H}{\delta \psi^{*}}, \quad H=\int\left\{\left|\nabla_{\perp} \psi\right|^{2}+\sigma\left|\psi_{x}\right|^{2}-\frac{\eta}{2}|\psi|^{4}\right\} d \mathbf{r} .
$$

In deriving Eq. (12.20) we have assumed that the kernel $T_{k_{1} k_{2} k_{3} k_{4}}$ is a continuous function of its arguments, that the vertex $T$ appearing in (12.9) is the value of this kernel at $\mathbf{k}_{i}=\mathbf{k}_{0}$. However, this situation is not typical, in particular if $\omega(0)=0$. At the same time, according to Goldstone's theorem (cf. [81]) the matrix element $V_{k k_{1} k_{2}}$ vanishes if one of the wave vectors $\mathbf{k}, \mathbf{k}_{1}$ or $\mathbf{k}_{2}$ is zero. Thus, in expression (12.9) for the matrix element of $T$, there are indeterminacies when $\mathbf{k}_{i}=\mathbf{k}_{0}$. To remove them we must calculate a limit of the type

$$
\lim \frac{\left|V_{k_{0}, k_{0}+k,-k}\right|^{2}}{\omega(k)-\mathbf{k v}_{g}}
$$

For example, for surface waves of infinite depth

$$
V_{k k_{0} k_{0}} \sim k^{3 / 4}, \omega(k) \sim k^{1 / 2}
$$

and all the indeterminacies vanish. For finite depth one has $V_{k k_{0} k_{0}} \sim k^{1 / 2}, \omega(k) \sim k$, so that this limit is finite in each direction, while the quantity $T_{k_{0} k_{0} k_{0} k_{0}}$ remains undetermined. Indeterminacy of this type is related to the excitation of forced motion of the medium as a whole. Such a situation occurs for all waves whose dispersion laws $\omega_{k}$ become linear as $k \rightarrow 0$. In addition to the surface waves considered above, such waves include ion-acoustic waves in plasma, sound waves in a solid, etc.

In this situation one needs separate equations for describing induced low-frequency motions. This problem is a special case of a more general question: the interaction of a spectrally narrow high-frequency wave packet with a low-frequency oscillation of acoustic type. The Hamiltonian for such an interaction can be constructed from general principles, based on the classical notion of an adiabatic invariant. (Of course, 
there is also a direct method of calculation, based on the reduction of the Hamiltonian (12.13)). We recall that for an oscillator of frequency $\omega$ there is the following remarkable relation between the energy $E$ and the adiabatic invariant $I$ :

$$
\frac{E}{\omega}=I
$$

In the present case the adiabatic invariant is the quantity $|c(k)|^{2}$, so that

$$
H_{0} \approx \omega\left(k_{0}\right) \int|c(k)|^{2} d \mathbf{k}=\int \omega\left(k_{0}\right)|\psi|^{2} d \mathbf{r} .
$$

A nonlinear interaction with low-frequency motions does not destroy the adiabaticity, so

$$
H_{\text {int }}=\int \delta \omega|\psi|^{2} d \mathbf{r}
$$

where $\delta \omega$ is the change in frequency of variations of the local characteristics of the medium, namely, the density $\delta \rho$ and velocity $\mathbf{v}$ :

$$
\delta \omega=\frac{\partial \omega}{\partial \rho_{0}} \delta \rho+\mathbf{k}_{0} \mathbf{v}
$$

(the second term corresponding to the Doppler effect).

Setting $\mathbf{v}=\nabla \varphi$ and remembering that $\delta \rho$ and $\varphi$ are canonically conjugate functions for a compressible fluid, we get the equations $[67,82]$ :

$$
\begin{gathered}
i\left(\psi_{t}+\mathbf{v}_{g} \nabla \psi\right)+\frac{\omega_{\alpha \beta}}{2} \frac{\partial^{2} \psi}{\partial x_{\alpha} \partial x_{\beta}}+\left(\frac{\partial \omega}{\partial \rho_{i}} \delta \rho+\mathbf{k}_{0} \nabla \varphi\right) \psi+\frac{T}{(2 \pi)^{3}}|\psi|^{2} \psi=0 \\
\frac{\partial}{\partial t} \delta \rho+\rho_{0} \Delta \varphi+\left(\mathbf{k}_{0} \nabla\right)|\psi|^{2}=0 \\
\rho_{0} \frac{\partial \varphi}{\partial t}+c_{s}^{2} \delta \rho+\frac{\partial \omega}{\partial \rho_{0}}|\psi|^{2}=0
\end{gathered}
$$

where $T$ is the regular part of the matrix element $\tilde{T}_{k_{0} k_{0} k_{0} k_{0}}$, having no singularities.

The Hamiltonian of this system is a combination of the Hamiltonians for Eqs. (12.20) and (4.3):

$$
\begin{gathered}
H_{c}=\int\left[-i \psi^{*} \mathbf{v}_{g} \nabla \psi+\frac{1}{2} \omega_{\alpha \beta} \frac{\partial \psi}{\partial x_{\alpha}} \frac{\partial \psi^{*}}{\partial x_{\beta}}+\left(\frac{\partial \omega}{\partial \rho_{0}} \delta \rho+\mathbf{k}_{0} \nabla \varphi\right)|\psi|^{2}\right. \\
\left.+\frac{1}{2} \frac{T}{(2 \pi)^{3}}|\psi|^{4}+c_{s}^{2} \frac{\delta \rho^{2}}{2 \rho_{0}}+\rho_{0} \frac{(\nabla \varphi)^{2}}{2}\right] d \mathbf{r} .
\end{gathered}
$$

Depending on the ratio between the group velocity $v_{g}$ and the sound velocity $c_{s}$, Eqs. (12.29), (12.30) permit various simplifications. If $v_{g}<c_{s}$ and $\epsilon_{g} \Delta k \gg T|\psi|^{2}$, 
where $\Delta k$ is the width around $k$ of the high-frequency packet, we can replace $\partial / \partial t$ by $\mathbf{v}_{g} \nabla$ in Eqs. (12.29), (12.30):

$$
\begin{aligned}
& -\mathbf{v}_{g} \nabla \delta \rho+\rho_{0} \Delta \varphi+\left(\mathbf{k}_{0} \nabla\right)|\psi|^{2}=0, \\
& -\rho_{0}\left(\mathbf{v}_{g} \nabla\right) \varphi+c_{s}^{2} \delta \rho+\frac{\partial \omega}{\partial \rho_{0}}|\psi|^{2}=0 .
\end{aligned}
$$

For isotropic media the resulting system of equations described in a coordinate system moving with the group velocity, goes over into the Davey-Stewartson equations

$$
\begin{gathered}
i \psi_{t}+\frac{v_{g}}{2 k_{0}} \Delta_{\perp} \psi+\frac{\omega^{\prime \prime}}{2} \psi_{x x}+\left[\frac{\partial \omega}{\partial \rho_{0}}+\frac{k_{0} c_{s}^{2}}{\rho_{0} v_{g}}\right] \delta \rho \psi \\
+\left[T /(2 \pi)^{3}+\frac{k_{0}}{\rho_{0} v_{g}} \frac{\partial \omega}{\partial \rho_{0}}\right]|\psi|^{2} \psi=0, \\
\left(v_{g} \frac{\partial}{\partial x}\right)^{2}\left(\delta \rho-\frac{k_{0}}{v_{g}}|\psi|^{2}\right)=\Delta\left(c_{s}^{2} \delta \rho+\frac{\partial \omega}{\partial \rho_{0}}|\psi|^{2}\right),
\end{gathered}
$$

which were first obtained for gravitational waves on the surface of a fluid of finite depth [83].

In this system Eq. (12.32) or (12.34) represents a constraint among $\delta \rho, \varphi$ and $|\psi|^{2}$, and the Hamiltonian for (12.33) is constructed taking these constraints into account. An explicit expression for it is easily obtained if we represent the constraint equations in the form

$$
-\left(\mathbf{v}_{g} \nabla\right) \delta \rho=\frac{\delta H}{\delta \varphi},-\left(\mathbf{v}_{g} \nabla\right) \varphi=\frac{\delta H}{\delta \rho}
$$

with $H=H_{c}$ (12.31). Then the Hamiltonian for the Davey-Stewartson equation $H_{D S}$ is gotten from $H_{c}$ by the following rule:

$$
H_{D S}=H_{c}-\int \delta \rho\left(\mathbf{v}_{g} \nabla\right) \varphi d \mathbf{r}+i \int \psi^{*}\left(\mathbf{v}_{g} \nabla\right) \psi d \mathbf{r}
$$

and the equations have the form

$$
i \psi_{t}=\frac{\delta H_{D S}}{\delta \psi^{*}}
$$

If $v_{g}>c_{s}$, then in Eqs. (12.29), (12.30), we cannot replace $\partial / \partial t$ by the operator $-\mathbf{v}_{g} \nabla$ whatever the level of nonlinearity may be. This is easily understood if we rewrite Eqs. (12.29), (12.30) in Fourier representation. If we carry this out, we are confronted by a resonance denominator of the form 


$$
k c_{s}=\left(\mathbf{k v}_{g}\right),
$$

which corresponds to the condition (12.7) for the decay of a high-frequency wave into high-frequency and sound waves. Under the condition $v_{g} \gg c_{s}$, corresponding, for example, to the interaction of light and sound in dielectrics, the contribution to $\delta \omega$ because of the Doppler effect is weak compared to the scattering by long-wave fluctuations of the density $\delta \rho$ of order $c_{s} / v_{g}$. In this case Eqs. (12.28)-(12.30) simplify to the form:

$$
\begin{gathered}
i \psi_{t}+\frac{\omega^{\prime \prime}}{2} \psi_{x x}+\frac{v_{g}}{2 k_{0}} \Delta_{\perp} \psi+\left(\frac{\partial \omega}{\partial \rho} \delta \rho+\frac{T}{(2 \pi)^{3}}|\psi|^{2}\right) \psi=0, \\
{\left[\left(\frac{\partial}{\partial t}-v_{g} \frac{\partial}{\partial x}\right)^{2}-c_{s}^{2} \Delta_{\perp}\right] \delta \rho=\frac{\partial \omega}{\partial \rho_{0}} \Delta|\psi|^{2} .}
\end{gathered}
$$

Among the simplest reductions one should also include the reduction of the Boussinesq equation to the KdV equation. For the Boussinesq model the dispersion law is close to linear. This means that in the Hamiltonian $H_{1}$ with coefficients of the form (4.5), one should keep the terms proportional to $a^{*} a a$ and $a a^{*} a^{*}$, and eliminate the other terms by canonical transformations, while in the quadratic Hamiltonian, we can keep in $\omega(k)$ the term linear in the dispersion $\nu: \omega(k)=k c_{s}\left(1+\left(\nu \rho_{0} k^{2} / 2 c_{s}^{2}\right)\right)$; then changing from the variables $a_{k}$ to $u(x)$ according to the formulas:

$$
a_{k}=\frac{u_{k}}{\sqrt{k}}, u=\int_{0}^{\infty}\left(u_{k} e^{i k x}+u_{k}^{*} e^{-i k x}\right) d k
$$

we then arrive at the $\mathrm{KdV}$ equation:

$$
u_{t}+c_{s} u_{x}+\beta u u_{x}+c_{s} \gamma u_{x x x}=0
$$

where

$$
\gamma=-\frac{\nu \rho_{0}}{2 c_{s}^{2}}, \beta=\frac{1}{2}\left(\frac{c_{s}}{\rho_{0}}\right)^{1 / 2}(1+g) .
$$

The natural generalization of the KDV equation to many dimensions is the KadomtsevPetviashvili (KP) equation [87] which follows if one considers the reduction of the Hamiltonian (4.5) to the case of the narrow angular distribution of the acoustic waves with a weak dispersion. Letting that the packet mainly propagates along the $x$-axis the equation (12.35) will transform into the form,

$$
\frac{\partial}{\partial x}\left(u_{t}+c_{s} u_{x}+\beta u u_{x}+c_{s} \gamma u_{x x x}\right)=\frac{c_{s}}{2} \nabla_{\perp}^{2} u,
$$

where the term in the left-hand side of the equation describes the diffraction of acoustic waves in the transverse direction to $x$. It is necessary to emphasize that 
all terms in this equation are small compared with respect to the second one, $c_{s} u_{x}$, responsible for the propagation along the $x$-axis of the packet with the sound velocity. And just in this sense the procedure of the derivation of the KP equation as well as the KDV equation represents one of the variants of the averaging methods when it is possible to distinguish two different temporal types of motion, rapid and slow.

The examples do not obviously exhaust all the possible reductions of Hamiltonians. We have only concentrated on the most bright ones, demonstrating their universality. A significant feature of this universality is that many of the models considered in this survey permit the application of the inverse scattering transform.

\section{Acknowledgements}

The authors thank Y.Pomeau, L.Bergé and V.V.Yankov for several useful remarks. One of us (E.K.) wishes to thank the Laboratoire de Physique Statistique of the Ecole Normale Superieur, where part of this work was performed, for its kind hospitality, and financial support through the Landau-CNRS agreement. This research was also supported in part by the INTAS, by the RFBR, and by the Russian State Program "Fundamental Problems of Nonlinear Dynamics".

\section{References}

[1] V.E.Zakharov and E.A.Kuznetsov, Sov. Sci. Rev., Ed. S.P.Novikov, 91, 1310 (1986).

[2] V.E.Zakharov and V.S.Lvov, Izv.Vuz.Radiofiz. 18, 1470 (1975).

[3] V.E.Zakharov, Izv.Vuz.Radiofiz. 17, 431 (1974).

[4] S.L.Musher, A.M.Rubenchik and V.E.Zakharov, Phys.Rep. 129, 285 (1985).

[5] G.B.Whitham, Linear and Nonlinear Waves (Wiley, New York, 1974).

[6] E.A.Kuznetsov, A.M.Rubenchik and V.E.Zakharov. Phys. Rep., 142, 103 (1986).

[7] D.Holm, J.Marsden, T.Ratiu and A.Weinstein, Phys. Rep., 123, 1-116, (1985).

[8] V.I.Arnold, Doklady Mat.Nauk, 162, 773-777 (1965).

[9] V.I.Arnold, Usp. Mat. Nauk 24, N3, 225 (1969).

[10] V.I.Arnold, Mathematical methods of classical mechanics, Moscow, Nauka, 1974. 
[11] V.I.Arnold, V.V.Kozlov and A.I.Neishtadt, in: "Itogi nauki i tekhniki" (ser. Sovremennye problemy matematiki), v. 3, Moscow, VINITI, 1985.

[12] L.D.Landau, JETP 11, 592 (1941).

[13] I.E.Dzyaloshinskii and G.E.Volovik, Ann.Phys. 125, 67 (1980).

[14] S.P.Novikov, Sov.Sci.Rev.,91 (1986).

[15] P.J.Morrison and J.M.Greene, Phys.Rev.Lett. 45, 790 (1980).

[16] P.J.Morrison, Phys.Lett. 80A, 383 (1980).

[17] H.Lamb, Hydrodynamics, Cambridge Univ. Press, 1932.

[18] E.A.Kuznetsov and A.V.Mikhailov, Phys.Lett. 77A, 37 (1980).

[19] H.Bateman, Proc. Royal Soc., A125, 598 (1929); Partial Differential Equations of Mathematical Physics, Cambridge Univ. Press, Cambridge (1944).

[20] D.I.Davydov, Dokl. Akad. Nauk SSSR 89:165 (1949).

[21] I.M.Khalatnikov, ZHETF (JETP) 23:169 (1952).

[22] R.L.Seliger, G.B.Whitham, Proc.Roy.Soc.Lond. A 305, 1 (1968).

[23] V.M.Kontorovich, H.Kravchik and V.Time, Hamiltonian description of nonpotential motion in the presence of a free surface in ordinary and magnetohydrodynamdics, Preprint 3-158, IRE, Acad.Sci,Ukr.SSR Kharkov, 1980.

[24] V.E.Zakharov and N.N.Filonenko, DAN USSR, 70, 1292 (1966).

[25] V.E.Zakharov, PMTF, N2, 86 (1968).

[26] A.G.Voronovich, Izv.Akad.Nauk SSSR, ser. fiz. atmosfer i okeana, 15, 82 (1979).

[27] V.P.Goncharov, Izv.Akad.Nauk SSSR, ser. fiz. atmosfer i okeana, 16, 473 (1980).

[28] Yu.Z.Miropolskii, Dynamics of Internal Gravitational Waves in the Ocean, Gidrometeoizdat, Leningrad, 1981.

[29] V.P.Goncharov and V.I.Pavlov, Problems of hydrodynamics in the Hamiltonian description, Moscow University Press, Moscow (1993).

[30] V.E.Zakharov, Physica, 3D, 193 (1981).

[31] V.E.Zakharov, Funk. Anal. i Prilozh. 14, N2, 15 (1980). 
[32] B.A.Kupershmidt and Yu.I.Manin, Funk. Anal. i Prilozh. 11, N3, 31 (1977); 12, N1, 25 (1978).

[33] V.E.Zakharov and E.A.Kuznetsov, DAN USSR, 194, 1288 (1970).

[34] V.E.Zakharov, ZHETF (JETP), 60, 1714 (1971).

[35] E.A.Kuznetsov, ZHETF (JETP), 62, 584 (1972).

[36] A.A.Kirillov, Elements of the Theory of Representations, Moscow, Nauka, 1978.

[37] B.Kostant, Proc. U.S.-Japan Seminar on Differential Geometry (Kyoto 1965), Tokyo, 1966.

[38] V.E.Zakharov, A.S.Monin and L.I.Piterbarg, Doklady Ac. Nauk SSSR, 289, 1061 (1987); V.E.Zakharov and L.I.Piterbarg, Doklady Ac. Nauk SSSR, 295, 86 (1987).

[39] L.I.Piterbarg, Phys. Lett. A 205, 149 (1995).

[40] V.E.Zakharov, S.V.Manakov, S.P.Novikov and L.P.Pitaevsky, Theory of solitons, Moscow, Nauka, 1980.

[41] R.Salmon,Hamilton's principle and Ertel's theorem, Am. Inst. Phys. Conf. Proc., 88, 127-135 (1982).

[42] H.Ertel, Meteorol. Z., 59, 277-281 (1942).

[43] R.Salmon,Hamiltonian fluid mechanics, Ann. Rev. Fluid Mech., 20, 225-256 (1988).

[44] C.Eckart, Phys. Rev., 54, 920 (1938); Phys. Fluids, 3, 421 (1960).

[45] C.Eckart, Phys Fluids, 6, 1037-1041 (1963).

[46] W.A.Newcomb, Proc. Symp. Appl. Math. 18, 152 (1967).

[47] N.Padhye and P.J.Morrison, Phys.Lett., A 219, 287 (1996).

[48] V.E.Zakharov and E.A.Kuznetsov, Hamiltonian formalism for systems of hydropdynamic type, Preprint no. 186, Int. Automation and Electrometry, Sib. Branch USSR Ac. Sci., Novosibirsk (1982).

[49] N.Padhye and P.J.Morrison, Phys.Lett., A 219, 287 (1996).

[50] A.H.Boozer, Magnetic field line Hamiltonian. Princeton Plasma Phys. Lab. Rep. No. PPPLR-2094R. 
[51] C.C.Lin, in Liquid Helium, Proceedings of the Enrico Fermi International School of Physics, Course XXI, Academic, New York, 1963.

[52] W.van Saarloos, Physica 108A, 557 (1981).

[53] L.D.Landau and E.M.Lifshits, Hydrodynamics, Moscow, Nauka (1988).

[54] L.D.Landau and E.M.Lifshits, Mechanics, Moscow, Nauka, 1965.

[55] J.J. Moreau, C.R.Acad. Sc. Paris, 252, 2810 (1961).

[56] H.K.Moffatt, J.Fluid Mech. 35, 117 (1969).

[57] V.V.Yankov, Pisma v ZHETF (JETP Letters), 58, 516 (1993); V.V.Yankov, ZHETF (JETP), 107, 414 (1995).

[58] L.Uby, M.B.Isichenko and V.V.Yankov, Phys.Rev.E, 52, 932 (1996).

[59] A.A.Abrashkin, D.A.Zen'kovich, E.I.Yakubovich, Izv. VUZ Radiofizika, XXXIX, 783 (1996).

[60] L.D.Faddeev, Lett.Math.Phys. 1, 289 (1976).

[61] B.A.Dubrovin, S.P.Novikov and A.T.Fomenko, Contemporary Geometry, Moscow, Nauka, 1979.

[62] J.H.C.Whitehead, Proc.Nat.Ac.Sci.USA 33, N5, 115 (1947).

[63] G.E.Volovik and V.P.Mineev, ZHETF (JETP), 72, 2256 (1977).

[64] H.D.I.Abarbanel, D.D.Holm, J.E.Marsden, and T.S.Ratiu, Philos. Trans. R.Soc. London Ser. A 318, 349-409 (1986).

[65] D.Lewis, J.Marsden and R.Montgomery, Physica 18D, 391-404, 1986.

[66] D.Ebin and J.Marsden, Ann. of Math., 92, 102-163 (1970).

[67] V.I.Karpman, Nonlinear Waves in Dispersive Media, Moscow, Nauka, 1973.

[68] E.A.Kuznetsov, M.D.Spector and V.E.Zakharov. Phys. Rev. E, 48, 1283 (1994).

[69] E.A.Kuznetsov and M.D.Spector, ZHETF (JETP), 71, 262 (1976).

[70] A.Rouhi and J.Wright, Phys. Rev. E, 48, 1850 (1993).

[71] V.P.Goncharov, Doklady Ac. Nauk SSSR, 313, 27 (1990).

[72] P.A.M.Dirac, Proc. Roy. Soc. A, 212, 330, (1952). 
[73] A.Frenkel, E.Levich, and L.Shtilman, Phys.Lett., 88A, 461 (1982).

[74] F.Henyey, A.I.P. Conf.Proc. 88, 85 (1982).

[75] V.A.Gordin and V.I.Petviashvili, Sov. J. Plasma Phys. 13, 509 (1987).

[76] S.S.Moiseev, R.Z.Sagdeev, A.V.Tur and V.V.Yanovskii, ZHETF (JETP), 83, 215 (1982).

[77] B.L.Gavrilin and M.M.Zaslavskii, DAN SSSR, bf 192, 48 (1982).

[78] V.E.Zakharov, Func. Anal. and Pril., 23, 24 (1989).

[79] M.A.Virasoro, Phys.Rev.Lett., 47, 1181 (1981).

[80] N.Bloembergen, Nonlinear Optics, Ed. W.A.Benjamin, Reading, Mass., 1965.

[81] N.N.Bogolyubov and D.V.Shirkov, Introduction to the Theory of Quantized Fields, Moscow, Nauka, 1976.

[82] V.E.Zakharov and A.M.Rubenchik, PMTF, N5, 84 (1972).

[83] A.Davey and K.Stewartson, Proc. Roy. Soc. London, A338,101 (1974).

[84] V.P.Goncharov, DAN SSSR, 313, 27 (1990).

[85] V.P.Krasitskii, ZHETF (JETP), 98, 1644 (1990).

[86] V.E.Zakharov, in Handbook of Plasma Physics, 2, ed.A.Galeeev and R.Sudan; (Elsevier, NY, 1984).

[87] B.B.Kadomtsev and V.I.Petviashvili. Dokl. Akad. Hauk SSSR, 192, 753 (1970) [Sov. Phys. Dokl. 15, 539 (1970)]. 